Synt het i c study di rected toward derivatives of bi ol ogi cal I y act i ve i ndol o[ 2, 3- a] car bazol e

\begin{tabular}{|l|l|}
\hline 著者 & $\begin{array}{l}\text { Sat o Nasako, Suzuki Yoshi aki, Yamada Fumi o, } \\
\text { Somei Nasanor i }\end{array}$ \\
\hline $\begin{array}{l}\text { j our nal or } \\
\text { publ i cat i on t i t l e }\end{array}$ & Het er ocycl es \\
\hline vol une & 80 \\
\hline number & 2 \\
\hline page r ange & $1027-1045$ \\
\hline year & $2010-01-01$ \\
\hline URL & ht t p: //hdl . handl e. net /2297/25289 \\
\hline
\end{tabular}


HETEROCYCLES, Vol. , No. , , pp. -,

Received, Accepted, , Published online, (Please do not delete.)

\title{
SYNTHETIC STUDY DIRECTED TOWARD DERIVATIVES OF BIOLOGICALLY ACTIVE INDOLO[2,3-a]CARBAZOLE ${ }^{\text {\# }}$
}

\author{
Masako Sato, Yoshiaki Suzuki, Fumio Yamada, and Masanori Somei** \\ Faculty of Pharmaceutical Sciences, Graduate School of Natural Science and \\ Technology, Kanazawa University, Kakuma-machi, Kanazawa, 920-1192, Japan \\ e-mail address: somei.home@ topaz.plala.or.jp
}

\begin{abstract}
Various derivatives of $\left(6 R^{*}, 6 \mathrm{a} R^{*}\right)$-6-chloro-6a-hydroxy-5,6,6a,12tetrahydroindolo[2,3-a]carbazole-5-one (8) and 6-cyano-5-hydroxyindolo[2,3$a$ carbazole (9) are prepared. Preparations of $\left(6 R^{*}, 6 \mathrm{a} R^{*}, 11 \mathrm{a} R^{*}\right)-6$-chloro-11acyano-6a-hydroxy- (11) and 12-substituted 6-(Z)-aminomethylidene5,6,6a,11,11a,12-hexahydroindolo[2,3-a]carbazole-5-ones (15) are also reported.
\end{abstract}

We have proposed a new concept for evaluating originality and efficiency of synthetic method introducing three measures such as originality rate, intellectual property factor, and application potential factor and defined an ideal synthetic method. ${ }^{2}$ In our continuing research, we have created a synthetic method, ${ }^{3}$ as one of the concrete example of the ideal synthesis, for 6-cyano-5-methoxy-12methylindolo[2,3-a]carbazole (1, Scheme 1) isolated from blue-green alga Nostoc sphaericum (strain EX5-1) by Moore and co-workers. ${ }^{4}$ The synthesis starts from indigo (2) and consists of six steps. Every compound involved in the synthesis has either a useful function or a biological activity. Thus, starting material is a widely used dye $\mathrm{e}^{5}$ and the target $\mathbf{1}$ is a cytotoxic and antiviral alkaloid. ${ }^{4}$ The compound $\mathbf{3}$ exhibits potent biological activity against telomerase. ${ }^{6}$ In addition, we have discovered as intellectual properties that 4,6 , and 7 are potent inhibitors of blood platelet aggregation ${ }^{7}$ while 5 is a promising $\alpha_{2}{ }^{-}$ blocker. $^{8}$

It is natural, therefore, that we would expect to discover a compound becoming medicine in the future among derivatives of biologically active $\mathbf{5}$ and $\mathbf{7}$. Now, we wish to report the synthesis of various deriva-

\footnotetext{
\# Dedicated to the $80^{\text {th }}$ birthday of Prof. Emeritus, Akira Suzuki, Hokkaido University.

$\ddagger$ Professor Emeritus of Kanazawa University. Present address: 56-7 Matsuhidai, Matsudo-shi, Chiba 270-2214, Japan.
} 
tives of 5 and 7. Interesting formations of $\left(6 R^{*}, 6 \mathrm{a} R^{*}, 11 \mathrm{a} R^{*}\right)-6$-chloro-11a-cyano-6a-hydroxy5,6,6a,11,11a,12-hexahydro- (11) and 12-substituted (Z)-6-aminomethylidene-5,6,11,12tetrahydroindolo[2,3- $a$ ]carbazole-5-one (15) are also reported.

\section{Scheme 1}

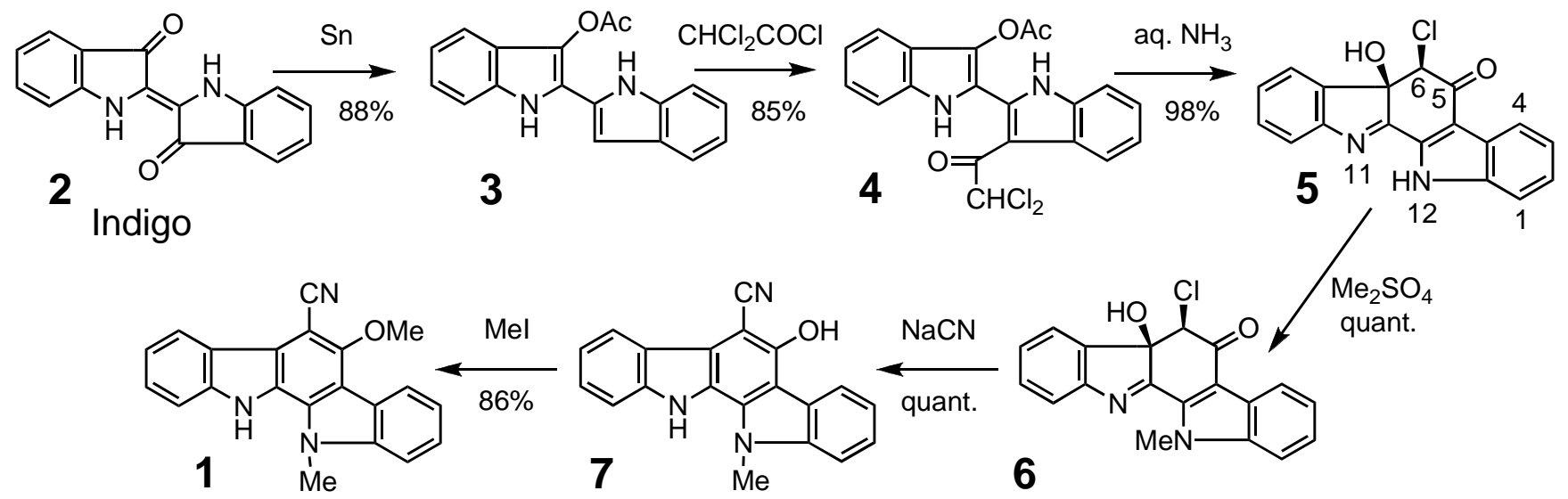

\section{Scheme 2}<smiles>[R]n1c2c(c3ccccc31)C(=O)C(Cl)C(O)(O)c1c-2n([R])c2ccc(CC)cc12</smiles>

8

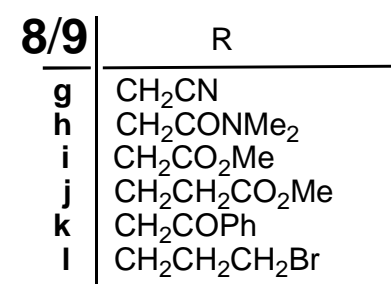<smiles>[R]n1c2ccccc2c2c(O)c(C=O)c3c4ccccc4[nH]c3c21</smiles>

14
$\mathrm{R}^{\prime}$

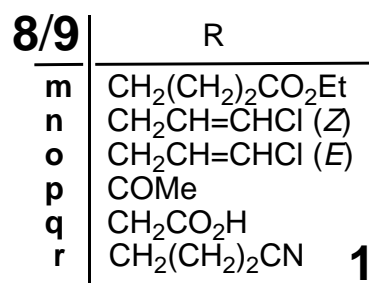

\begin{tabular}{c|cc}
12 & $R^{1}$ & $R^{2}$ \\
\hline $\mathbf{a}$ & $H$ & $H$ \\
$\mathbf{b}$ & $A c$ & $A c$
\end{tabular}
10<smiles>N#Cc1c(O)c2c3ccccc3[nH]c2c2[nH]c3ccccc3c12</smiles><smiles>N#CC12Nc3ccccc3C1(O)C(Cl)C(=O)c1ccccc12</smiles>

11<smiles>COc1c(C#N)c2c3ccccc3[nH]c2c2c1c1ccccc1n2C</smiles>

13

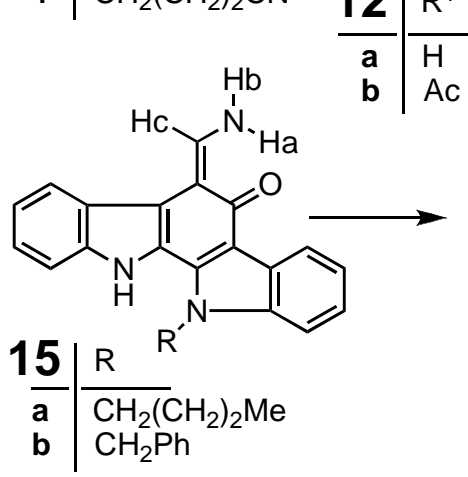

First, the compound 5 was prepared according to our procedures ${ }^{3}$ from indigo in three steps in $73 \%$ overall yield. Subsequent treatment of $\mathbf{5}$ in $N, N$-dimethylformamide (DMF) in the presence of $\mathrm{K}_{2} \mathrm{CO}_{3}$ with $n$-butyl iodide, allyl bromide, propargyl bromide, benzyl bromide, phenethyl bromide, and $(E)$ cinnamyl bromide provided $\mathbf{8 a}, \mathbf{8 b}, \mathbf{8 c}, \mathbf{8 d}, \mathbf{8 e}$, and $\mathbf{8 f}$ in the respective yields of $71,96,50,78,57$, and 96\% (Scheme 2). Similar treatment of 5 with reagents having a cyano or a carbonyl group such as 
chloroacetonitrile, $\mathrm{N}, \mathrm{N}$-dimethyl-2-chloroacetamide, methyl bromoacetate, methyl acrylate, and phenacyl bromide afforded $\mathbf{8 g}, \mathbf{8 h}, \mathbf{8 i}, \mathbf{8 j}$, and $\mathbf{8 k}$ in the respective yields of 62, 90, 72, 26, and 97\%. Although the reaction of 1,3-dibromopropane with $\mathbf{5}$ similarly proceeded to give $\mathbf{8 1}$ in $45 \%$ yield, ethyl 4-bromobutylate did not react with 5 at all. To overcome the problem, change of the base from $\mathrm{K}_{2} \mathrm{CO}_{3}$ to $\mathrm{NaH}$ in anhydrous DMF was successful to obtain the desired $8 \mathrm{~m}$ in $59 \%$ yield.

In the reaction of 5 with an $E, Z$ mixture of 1,3-dichloropropene, $\mathrm{NaH}$ in anhydrous DMF was the reaction conditions of choice, providing 12-(Z)- 8n and 12-(E)-(3-chloroallyl) derivatives 80 in 42 and 24\% yields, respectively. Under similar reaction conditions, 5 reacted with acetyl chloride to produce 12-acetyl compound $\mathbf{8 p}$ in $41 \%$ yield.

With various 12-substituted $\quad\left(6 R^{*}, 6 \mathrm{a} R^{*}\right)$-6-chloro-6a-hydroxy-5,6,6a,12-tetrahydroindolo[2,3a]carbazole-5-one in hand, we next employed our reductive cyanation which realized transformation of 6 to 7. Thus, the treatment of $\mathbf{8 a}$ with $\mathrm{NaCN}$ in $\mathrm{DMF}-\mathrm{H}_{2} \mathrm{O}$ provided 12- $n$-butyl-6-cyano-5hydroxyindolo[2,3-a]carbazole (9a) in 85\% yield. A possible reaction mechanism is shown in Scheme 3. The initial step would be a nucleophilic substitution for $6 \beta$-chloride by cyanide from the back side to give A. After dehydration, the resultant $\mathbf{B}$ can form $\mathbf{C}$ by general acid promoted cyanide addition to the 6position. Subsequent cyanide attack at one of the geminal cyano groups of $\mathbf{C}$ achieves the reductive cyanation with the liberation of dicyan and an enolate of 9a. The other possible route is the enolization of 5-carbonyl of $\mathbf{A}$, followed by the addition of cyanide at the imine carbon $\left(\mathrm{C}_{11 \mathrm{a}}\right)$ from the less sterically hindered $\alpha$-side culminating in the formation of $\mathbf{D}$. Subsequent cyanide attack at the $11 \alpha$-cyano group and concomitant general acid promoted elimination of 6aß-hydroxy group liberates dicyan and 9a.

\section{Scheme 3. Possible Mechanism}

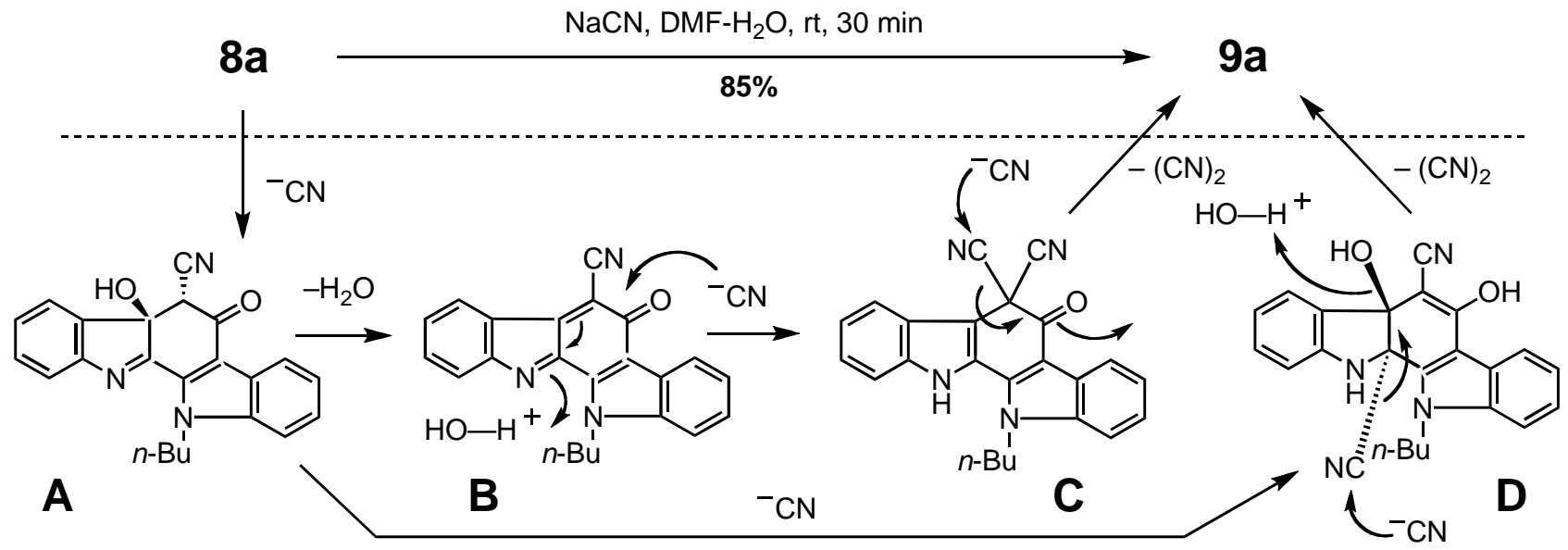

On the basis of above results, the reductive cyanation was applied to $\mathbf{8 b}-\mathbf{h}$ and $\mathbf{8 m}$ resulting in the formations of $\mathbf{9 b}, \mathbf{9 c}, \mathbf{9 d}, \mathbf{9 e}, \mathbf{9 f}, \mathbf{9 g}, \mathbf{9 h}$, and $\mathbf{9 m}$ in the respective yields of 91, 72, 98, 85, 80, 54, 95, and $72 \%$. In the case of $\mathbf{8 i}$, the reductive cyanation formed $\mathbf{9 i}$ and $\mathbf{9 q}$ in 17 and $43 \%$ yields, respectively. Similar reaction of $\mathbf{8 k}$ removed the 12-phenacyl group to afford $\mathbf{1 0}$ in $27 \%$ yield together with $16 \%$ yield of starting material. 
The structures of $\mathbf{9 a - i}$ and $\mathbf{9 m}, \mathbf{q}$ were established unequivocally by pursuing X-ray single-crystal analysis of 9a as a representative of them and the results are shown in Figure 1 and Table 1. It is interesting to note that this type of compounds 9 tends to involve a recrystallization solvent molecule in crystals. In fact, the ORTEP drawing of 9a demonstrates EtOAc molecule.

Figure 1. ORTEP Drawing of 9 a $(R=0.049)$

Figure 2. ORTEP Drawing of $11(R=0.085)$
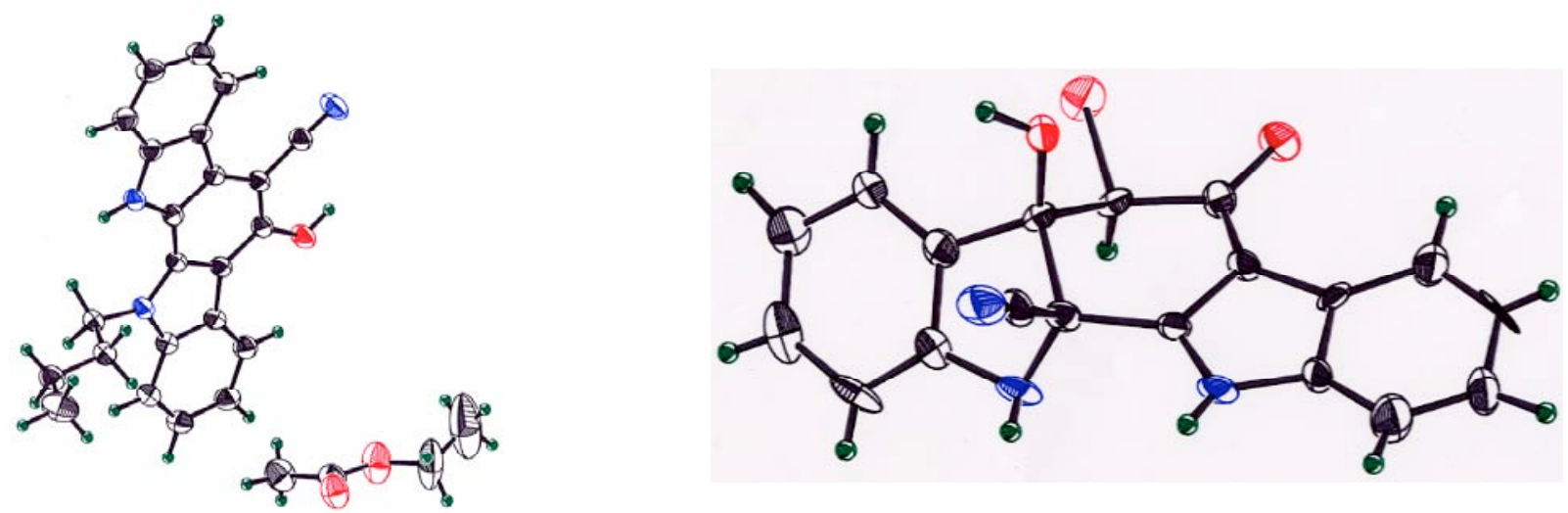

The reaction of $\mathbf{8 1}$ with $\mathrm{NaCN}$ in $\mathrm{DMF}-\mathrm{H}_{2} \mathrm{O}$ provided 91 and $9 \mathrm{r}$ in 23 and 23\% yields, respectively. The reaction of about 4:1 mixture of $8 \mathbf{n}$ and 80 produced $9 \mathbf{n}$ and 90 in 41 and 14\% yields, respectively. It is interesting to note that the similar reaction of $\mathbf{8 p}$ afforded $\mathbf{1 0}$ and the unexpected $\left(6 R^{*}, 6 \mathrm{a} R^{*}, 11 \mathrm{a} R^{*}\right)-6$ chloro-11a-cyano-6a-hydroxy-5,6,6a,11,11a,12-hexahydroindolo[2,3-a]carbazole-5-one (11) in 22 and $74 \%$ yields, respectively, though formation of the desired $\mathbf{9 p}$ was not observed at all.

Reduction of 11 with $\mathrm{NaBH}_{4}$ proceeded slowly from the less hindered $\beta$-side to provide $5 \alpha$-hydroxy compound 12a in $47 \%$ yield. Further treatment of $\mathbf{1 2 a}$ with acetic anhydride gave $5 \alpha$-acetoxy compound 12b in 30\% yield. Comparing ${ }^{1} \mathrm{H}-\mathrm{NMR}$ spectra of $\mathbf{1 2 a}$ and $\mathbf{1 2 b}$, the coupling constant between $\mathrm{H}_{5}$ and $\mathrm{H}_{6}$ is shown to be $8.2 \mathrm{~Hz}$, which proved their stereochemistries as shown in the Scheme 2.

It should be noted that the absorption bands of cyano group of 11, 12a, and 12b were very weak or almost invisible in their infrared spectra. Therefore, X-ray single-crystal analysis of $\mathbf{1 1}$ was necessary for the determination of the structure. The results shown in Figure 2 and Table 2 demonstrate both the presence of the cyano group at the 11a-position and the stereochemistries of 6, 6a, and 11a positions being all $R^{*}$. Methylation of 9d with ethereal diazomethane smoothly proceeded to afford the corresponding methoxy compound 13 in $82 \%$ yield. All attempts to hydrolyze the 6-cyano group of $\mathbf{1 3}$ to 6-carboxy or 6carbamoyl group with base were unsuccessful. Under severe conditions such as treatment of $\mathbf{1 3}$ with solid $\mathrm{NaOH}$ in refluxing ethylene glycol resulted in the methyl ether cleavage to afford $9 \mathbf{d}$ in $62 \%$ yield.

Further attempt to obtain 6-formyl type compound $\mathbf{1 4}$ by the reaction of 9 with diisobutyl aluminum hydride (DIBAL) proceeded in an unexpected way. Thus the reduction of 9a and 9d with DIBAL afforded 15a and 15b in 73 and 47\% yields, respectively. In the ${ }^{1} \mathrm{H}-\mathrm{NMR}$ spectrum of $\mathbf{1 5 a}$, hydrogen bonded Ha was observed at lower $\delta 12.0(1 \mathrm{H}, \mathrm{dd}, J=13.8,8.2 \mathrm{~Hz})$, while $\mathrm{Hb}$ and $\mathrm{Hc}$ protons appeared at 
$\delta 8.60(1 \mathrm{H}$, brt, $J=8.2 \mathrm{~Hz})$ and $8.84(1 \mathrm{H}, \mathrm{dd}, J=13.8,8.2 \mathrm{~Hz})$, respectively. On the addition of $\mathrm{D}_{2} \mathrm{O}$, both $\mathrm{Ha}$ and $\mathrm{Hb}$ protons disappeared and $\mathrm{Hc}$ collapsed to a singlet. Similar phenomena were observed in case of $\mathbf{1 5 b}$. Further treatment of $\mathbf{1 5 a}$ with $\mathrm{Ac}_{2} \mathrm{O}$ afforded $\mathrm{N}$-acetyl compound $\mathbf{1 6}$ in $87 \%$ yield. These results prove the 6-aminomethylidene structures of $\mathbf{1 5 a}, \mathbf{b}$.

A possible reaction mechanism for the transformation of $\mathbf{9}$ to $\mathbf{1 5}$ is shown in Scheme 4. The initial reaction of DIBAL with $\mathbf{9}$ forms aluminum complex $\mathbf{A}$, followed by the intramolecular reduction of cyano group with hydride to afford $\mathbf{B}$. Hydrolysis of $\mathbf{B}$ affords an enol form compound $\mathbf{C}$ which tautomerizes to a carbonyl form product $\mathbf{1 5}$ forming a stable enamide system.

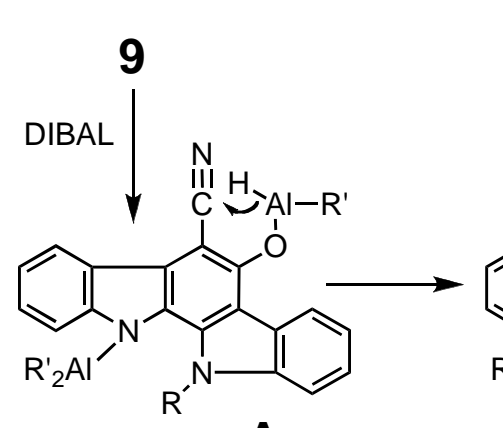

A
Scheme 4

$\mathrm{R}=\mathrm{CH}_{2}\left(\mathrm{CH}_{2}\right)_{2} \mathrm{Me}$ or $\mathrm{CH}_{2} \mathrm{Ph}$

In summary, we succeeded in preparing various derivatives of $\mathbf{8}$ and $\mathbf{9}$, together with new classes of compound, 11 and 15. Biological evaluations of new compounds in this report are in progress.

\section{EXPERIMENTAL}

Melting points were determined on a Yanagimoto micro melting point apparatus and are uncorrected. Infrared (IR) spectra were recorded with a Shimadzu IR-420 or Horiba FT-720 spectrophotometer and proton nuclear magnetic resonance $\left({ }^{1} \mathrm{H}-\mathrm{NMR}\right)$ spectra with a JEOL GSX-500 spectrometer with tetramethylsilane as an internal standard. Mass spectra (MS) were recorded on a JEOL JMS-SX102A instruments. Column chromatography was performed on silica gel $\left(\mathrm{SiO}_{2}, 100-200\right.$ mesh, from Kanto Chemical Co., Inc.) throughout the present study.

$\left(6 R^{*}, 6 \mathrm{a} R^{*}\right)-12-n$-Butyl-6-chloro-6a-hydroxy-5,6,6a,12-tetrahydroindolo[2,3-a]carbazole-5-one

from $\left(6 R^{*}, 6 \mathrm{a} R^{*}\right)-6$-Chloro-6a-hydroxy-5,6,6a,12-tetrahydroindolo[2,3-a]carbazole-5-one $\quad(5)$

General Procedure A: $\mathrm{K}_{2} \mathrm{CO}_{3}(72.1 \mathrm{mg}, 0.52 \mathrm{mmol})$ and $n$-butyl iodide $(0.34 \mathrm{~mL}, 2.90 \mathrm{mmol})$ were added to a solution of $5(48.1 \mathrm{mg}, 0.15 \mathrm{mmol})$ in DMF $(3.0 \mathrm{~mL})$, and the mixture was stirred for $20 \mathrm{~min}$ at rt. After addition of $\mathrm{H}_{2} \mathrm{O}$ under ice cooling, the whole was extracted with EtOAc. The extract was washed with brine, dried over $\mathrm{Na}_{2} \mathrm{SO}_{4}$, and evaporated under reduced pressure to leave an oil, which was column-chromatographed on $\mathrm{SiO}_{2}$ with EtOAc-hexane (1:5, v/v) to give 8a $(40.1 \mathrm{mg}, 71 \%)$. 8a: $\mathrm{mp}$ $187-189^{\circ} \mathrm{C}$ (decomp., orange prisms, recrystallized from $\mathrm{CHCl}_{3}$ ). IR (KBr): 3421, 1653, 1479, 1346, 1086, $754 \mathrm{~cm}^{-1} .{ }^{1} \mathrm{H}-\mathrm{NMR}\left(\mathrm{CDCl}_{3}\right)$ $\delta: 0.97(3 \mathrm{H}, \mathrm{t}, J=7.4 \mathrm{~Hz}), 1.39-1.50(2 \mathrm{H}, \mathrm{m}), 1.87-2.00(2 \mathrm{H}, \mathrm{m})$, 
$3.11\left(1 \mathrm{H}, \mathrm{s}\right.$, disappeared on addition of $\left.\mathrm{D}_{2} \mathrm{O}\right), 4.63(1 \mathrm{H}, \mathrm{ddd}, J=14.1,8.2,6.4 \mathrm{~Hz}), 4.76(1 \mathrm{H}, \mathrm{s}), 4.87(1 \mathrm{H}$, ddd, $J=14.1,8.2,6.4 \mathrm{~Hz}), 7.35(1 \mathrm{H}, \mathrm{td}, J=7.7,1.2 \mathrm{~Hz}), 7.39(1 \mathrm{H}, \mathrm{td}, J=7.7,1.2 \mathrm{~Hz}), 7.47(1 \mathrm{H}, \mathrm{td}, J=7.7$, $1.2 \mathrm{~Hz}), 7.50(1 \mathrm{H}, \mathrm{d}, J=7.7 \mathrm{~Hz}), 7.50(1 \mathrm{H}, \mathrm{td}, J=7.7,1.2 \mathrm{~Hz}), 7.72(1 \mathrm{H}, \mathrm{d}, J=7.7 \mathrm{~Hz}), 7.92(1 \mathrm{H}, \mathrm{d}, J=7.7$ $\mathrm{Hz}), 8.39(1 \mathrm{H}, \mathrm{d}, J=7.7 \mathrm{~Hz})$. MS $m / z: 380$ and $378\left(\mathrm{M}^{+}\right)$. Anal. Calcd for $\mathrm{C}_{22} \mathrm{H}_{19} \mathrm{~N}_{2} \mathrm{O}_{2} \mathrm{Cl} \cdot 1 / 8 \mathrm{H}_{2} \mathrm{O}: \mathrm{C}, 69.33$; H, 5.09; N, 7.35. Found: C, 69.38; H, 5.11; N, 7.24.

$\left(6 R^{*}, 6 \mathrm{a} R^{*}\right)$-12-Allyl-6-chloro-6a-hydroxy-5,6,6a,12-tetrahydroindolo[2,3-a]carbazole-5-one

from 5 - In the general procedure $\mathrm{A}, \mathrm{K}_{2} \mathrm{CO}_{3}(754 \mathrm{mg}, 5.44 \mathrm{mmol})$, allyl bromide $(2.70 \mathrm{~mL}, 31.1 \mathrm{mmol})$, 5 (501 mg, $1.56 \mathrm{mmol})$, and DMF (10.0 mL) were used. The reaction time was $30 \mathrm{~min}$. After columnchromatography, 8b (540 mg, 96\%) was obtained. 8b: mp 202-203 $\mathrm{C}$ (decomp., yellow prisms, recrystallized from EtOAc). IR (KBr): 3400, 3110, 1665, 1562, 1457, 1333, 1140, 1087, 1017, 789, 747 $\mathrm{cm}^{-1} .{ }^{1} \mathrm{H}-\mathrm{NMR}\left(\mathrm{DMSO}-d_{6}\right)$ d: $5.16(1 \mathrm{H}, \mathrm{dd}, J=17.1,1.5 \mathrm{~Hz}), 5.22(1 \mathrm{H}, \mathrm{dd}, J=10.3,1.5 \mathrm{~Hz}), 5.35(1 \mathrm{H}, \mathrm{s})$, $5.36(1 \mathrm{H}, \mathrm{dd}, J=16.3,5.4 \mathrm{~Hz}), 5.53(1 \mathrm{H}, \mathrm{dd}, J=16.3,5.4 \mathrm{~Hz}), 6.07-6.16(1 \mathrm{H}, \mathrm{m}), 6.84(1 \mathrm{H}, \mathrm{s}), 7.39(1 \mathrm{H}$, t, $J=8.1 \mathrm{~Hz}), 7.40(1 \mathrm{H}, \mathrm{t}, J=8.1 \mathrm{~Hz}), 7.50(1 \mathrm{H}, \mathrm{t}, J=7.5 \mathrm{~Hz}), 7.55(1 \mathrm{H}, \mathrm{t}, J=7.5 \mathrm{~Hz}), 7.75(1 \mathrm{H}, \mathrm{d}, J=8.1$ Hz), $7.77(1 \mathrm{H}, \mathrm{d}, J=7.5 \mathrm{~Hz}), 7.84(1 \mathrm{H}, \mathrm{d}, J=7.5 \mathrm{~Hz}), 8.19(1 \mathrm{H}, \mathrm{d}, J=8.1 \mathrm{~Hz}) . \mathrm{MS} m / z: 364$ and $362\left(\mathrm{M}^{+}\right)$. Anal. Calcd for $\mathrm{C}_{21} \mathrm{H}_{15} \mathrm{~N}_{2} \mathrm{O}_{2} \mathrm{Cl}$ : C, 69.52; H, 4.17; N, 7.72. Found: C, 69.49; H, 4.17; N, 7.41.

$\left(6 R^{*}, 6 a R^{*}\right)-6$-Chloro-6a-hydroxy-12-propargyl-5,6,6a,12-tetrahydroindolo[2,3-a]carbazole-5-one

(8c) from 5 - In the general procedure $\mathrm{A}, \mathrm{K}_{2} \mathrm{CO}_{3}(44.2 \mathrm{mg}, 0.32 \mathrm{mmol})$, propargyl bromide $(0.14 \mathrm{~mL}$, $1.83 \mathrm{mmol}), 5(29.5 \mathrm{mg}, 0.09 \mathrm{mmol})$, and DMF $(2.0 \mathrm{~mL})$ were used. The reaction time was $1 \mathrm{~h}$. After column-chromatography, 8c $(16.5 \mathrm{mg}, 50 \%)$ was obtained. 8c: $\mathrm{mp} 248^{\circ} \mathrm{C}$ (decomp., dark yellow powder, recrystallized from EtOAc-hexane). IR (KBr): 3359, 3286, 1653, 1475, 1086, 791, $746 \mathrm{~cm}^{-1} .{ }^{1} \mathrm{H}-\mathrm{NMR}$ $\left(\mathrm{DMSO}-d_{6}\right) \delta: 3.48(1 \mathrm{H}, \mathrm{t}, J=2.5 \mathrm{~Hz}), 5.38\left(1 \mathrm{H}, \mathrm{d}, J=1.5 \mathrm{~Hz}\right.$, collapsed to s on addition of $\left.\mathrm{D}_{2} \mathrm{O}\right), 5.66(1 \mathrm{H}$, $\mathrm{dd}, J=17.9,2.5 \mathrm{~Hz}), 5.78(1 \mathrm{H}, \mathrm{dd}, J=17.9,2.5 \mathrm{~Hz}), 6.91(1 \mathrm{H}, \mathrm{d}, J=1.5 \mathrm{~Hz}$, disappeared on addition of $\left.\mathrm{D}_{2} \mathrm{O}\right), 7.41(1 \mathrm{H}, \mathrm{td}, J=7.7,1.3 \mathrm{~Hz}), 7.45(1 \mathrm{H}, \mathrm{td}, J=7.7,1.3 \mathrm{~Hz}), 7.56(1 \mathrm{H}, \mathrm{td}, J=7.7,1.3 \mathrm{~Hz}), 7.56(1 \mathrm{H}, \mathrm{td}$, $J=7.7,1.3 \mathrm{~Hz}), 7.81(1 \mathrm{H}, \mathrm{d}, J=7.7 \mathrm{~Hz}), 7.84(1 \mathrm{H}, \mathrm{d}, J=7.7 \mathrm{~Hz}), 7.84(1 \mathrm{H}, \mathrm{d}, J=7.7 \mathrm{~Hz}), 8.19(1 \mathrm{H}, \mathrm{d}$, $J=7.7 \mathrm{~Hz})$. MS m/z: 362 and $360\left(\mathrm{M}^{+}\right)$. Anal. Calcd for $\mathrm{C}_{21} \mathrm{H}_{13} \mathrm{~N}_{2} \mathrm{O}_{2} \mathrm{Cl} \cdot 1 / 2 \mathrm{H}_{2} \mathrm{O}: \mathrm{C}, 68.21 ; \mathrm{H}, 3.82 ; \mathrm{N}, 7.58$. Found: C, 68.00; H, 3.73; N, 7.33.

$\left(6 R^{*}, 6 a R^{*}\right)-12-B e n z y l-6-c h l o r o-6 a-h y d r o x y-5,6,6 a, 12-t e t r a h y d r o i n d o l o[2,3-a]$ carbazole-5-one

from 5 - In the general procedure $\mathrm{A}, \mathrm{K}_{2} \mathrm{CO}_{3}(619 \mathrm{mg}, 4.48 \mathrm{mmol})$, benzyl bromide $(3.10 \mathrm{~mL}, 25.6$ mmol), 5 (413 mg, $1.28 \mathrm{mmol})$, and DMF $(8.0 \mathrm{~mL})$ were used. The reaction time was $75 \mathrm{~min}$. After column-chromatography, 8d (411 mg, 78\%) was obtained. 8d: $\mathrm{mp} 219.5-221.5{ }^{\circ} \mathrm{C}$ (yellow prisms, recrystallized from EtOAc-hexane). IR (KBr): 3356, 1685, 1577, 1473, 1142, $771 \mathrm{~cm}^{-1} .{ }^{1} \mathrm{H}-\mathrm{NMR}$ $\left(\mathrm{DMSO}-d_{6}\right)$ d: $5.41(1 \mathrm{H}, \mathrm{s}), 5.99(1 \mathrm{H}, \mathrm{d}, J=15.8 \mathrm{~Hz}), 6.18(1 \mathrm{H}, \mathrm{d}, J=15.8 \mathrm{~Hz}), 6.91(1 \mathrm{H}$, br s, disappeared on addition of $\left.\mathrm{D}_{2} \mathrm{O}\right), 7.26(1 \mathrm{H}, \mathrm{t}, J=7.2 \mathrm{~Hz}), 7.30(2 \mathrm{H}, \mathrm{t}, J=7.2 \mathrm{~Hz}), 7.37(2 \mathrm{H}, \mathrm{d}, J=7.2 \mathrm{~Hz}), 7.40(1 \mathrm{H}, \mathrm{td}$, $J=7.4,1.2 \mathrm{~Hz}), 7.42(1 \mathrm{H}, \mathrm{td}, J=7.4,1.2 \mathrm{~Hz}), 7.42(1 \mathrm{H}, \mathrm{td}, J=7.4,1.2 \mathrm{~Hz}), 7.54(1 \mathrm{H}, \mathrm{td}, J=7.4,1.2 \mathrm{~Hz})$, 
$7.64(1 \mathrm{H}, \mathrm{d}, J=7.4 \mathrm{~Hz}), 7.75(1 \mathrm{H}, \mathrm{d}, J=7.4 \mathrm{~Hz}), 7.86(1 \mathrm{H}, \mathrm{d}, J=7.4 \mathrm{~Hz}), 8.19(1 \mathrm{H}, \mathrm{d}, J=7.4 \mathrm{~Hz}) . \mathrm{MS} m / z$ : 414 and $412\left(\mathrm{M}^{+}\right)$. Anal. Calcd for $\mathrm{C}_{25} \mathrm{H}_{17} \mathrm{~N}_{2} \mathrm{O}_{2} \mathrm{Cl} \cdot 1 / 2 \mathrm{H}_{2} \mathrm{O}: \mathrm{C}, 71.17 ; \mathrm{H}, 4.30 ; \mathrm{N}, 6.64$. Found: C, 71.32; $\mathrm{H}$, $4.25 ; \mathrm{N}, 6.49$.

$\left(6 R^{*}, 6 a R^{*}\right)-6$-Chloro-6a-hydroxy-12-phenethyl-5,6,6a,12-tetrahydroindolo[2,3-a]carbazole-5-one

(8e) from 5 - In the general procedure $\mathrm{A}, \mathrm{K}_{2} \mathrm{CO}_{3}(45.0 \mathrm{mg}, 0.33 \mathrm{mmol})$, phenethyl bromide $(0.25 \mathrm{~mL}$, $1.86 \mathrm{mmol}), 5(30.0 \mathrm{mg}, 0.09 \mathrm{mmol})$, and DMF (2.0 mL) were used. The reaction time was $1 \mathrm{~h}$. After column-chromatography, 8e (22.6 mg, 57\%) was obtained. 8e: mp 183-184 ${ }^{\circ} \mathrm{C}$ (yellow needles, recrystallized from EtOAc-hexane). IR (KBr): 3361, 1655, 1477, 1146, $746 \mathrm{~cm}^{-1} .{ }^{1} \mathrm{H}-\mathrm{NMR}$ (DMSO- $d_{6}$ ) $\delta$ : $3.19(2 \mathrm{H}, \mathrm{td}, J=13.8,6.8 \mathrm{~Hz}), 4.83(1 \mathrm{H}, \mathrm{ddd}, J=13.8,8.6,6.8 \mathrm{~Hz}), 5.04$ (1H, ddd, $J=13.8,8.6,6.8 \mathrm{~Hz})$, $5.36(1 \mathrm{H}, \mathrm{s}), 6.84\left(1 \mathrm{H}\right.$, br s, disappeared on addition of $\left.\mathrm{D}_{2} \mathrm{O}\right), 7.21(1 \mathrm{H}$, br t,$J=7.4 \mathrm{~Hz}), 7.30(2 \mathrm{H}, \mathrm{t}, J=7.4$ $\mathrm{Hz}), 7.35(2 \mathrm{H}, \mathrm{d}, J=7.4 \mathrm{~Hz}), 7.37(1 \mathrm{H}, \mathrm{t}, J=7.5 \mathrm{~Hz}), 7.41(1 \mathrm{H}, \mathrm{t}, J=7.5 \mathrm{~Hz}), 7.43(1 \mathrm{H}, \mathrm{t}, J=7.5 \mathrm{~Hz}), 7.58$ $(1 \mathrm{H}, \mathrm{t}, J=7.5 \mathrm{~Hz}), 7.73(1 \mathrm{H}, \mathrm{d}, J=7.5 \mathrm{~Hz}), 7.84(1 \mathrm{H}, \mathrm{d}, J=7.5 \mathrm{~Hz}), 7.86(1 \mathrm{H}, \mathrm{d}, J=7.5 \mathrm{~Hz}), 8.15(1 \mathrm{H}, \mathrm{d}$, $J=7.5 \mathrm{~Hz})$. MS m/z: 428 and $426\left(\mathrm{M}^{+}\right)$. Anal. Calcd for $\mathrm{C}_{26} \mathrm{H}_{19} \mathrm{~N}_{2} \mathrm{O}_{2} \mathrm{Cl} \cdot 1 / 2 \mathrm{H}_{2} \mathrm{O}: \mathrm{C}, 71.64 ; \mathrm{H}, 4.62 ; \mathrm{N}, 6.43$. Found: C, 71.86; H, 4.43; N, 6.40 .

$\left(6 R^{*}, 6 a R^{*}\right)-6$-Chloro-12-(E)-cinnamyl-6a-hydroxy-5,6,6a,12-tetrahydroindolo[2,3-a]carbazole-5one (8f) from 5 - In the general procedure $\mathrm{A}, \mathrm{K}_{2} \mathrm{CO}_{3}(157 \mathrm{mg}, 1.14 \mathrm{mmol})$, cinnamyl bromide $(0.79 \mathrm{~mL}$, $6.49 \mathrm{mmol}), 5(105 \mathrm{mg}, 0.32 \mathrm{mmol})$, and DMF $(6.0 \mathrm{~mL})$ were used. The reaction time was $1 \mathrm{~h}$. After column-chromatography, 8f (137 mg, 96\%) was obtained. 8f: mp 205-208 $\mathrm{C}$ (brown plates, recrystallized from EtOAc-hexane). IR (KBr): 3390, 1648, 1579, 1473, 1145, $757 \mathrm{~cm}^{-1} .{ }^{1} \mathrm{H}-\mathrm{NMR}$ $\left(\mathrm{DMSO}-d_{6}\right) \delta: 5.36\left(1 \mathrm{H}, \mathrm{d}, J=1.5 \mathrm{~Hz}\right.$, collapsed to s on addition of $\left.\mathrm{D}_{2} \mathrm{O}\right), 5.51(1 \mathrm{H}, \mathrm{ddd}, J=16.5,5.9,1.2$ Hz), $5.71(1 \mathrm{H}, \mathrm{ddd}, J=16.5,5.9,1.2 \mathrm{~Hz}), 6.53(1 \mathrm{H}, \mathrm{dt}, J=16.5,5.9 \mathrm{~Hz}), 6.69(1 \mathrm{H}, \mathrm{d}, J=16.5 \mathrm{~Hz}), 6.85$ $\left(1 \mathrm{H}, \mathrm{d}, J=1.5 \mathrm{~Hz}\right.$, disappeared on addition of $\left.\mathrm{D}_{2} \mathrm{O}\right), 7.22(1 \mathrm{H}, \mathrm{t}, J=7.4 \mathrm{~Hz}), 7.28(2 \mathrm{H}, \mathrm{t}, J=7.4 \mathrm{~Hz}), 7.37$ $(2 \mathrm{H}, \mathrm{d}, J=7.4 \mathrm{~Hz}), 7.40(1 \mathrm{H}, \mathrm{td}, J=7.6,1.2 \mathrm{~Hz}), 7.41(1 \mathrm{H}, \mathrm{td}, J=7.6,1.2 \mathrm{~Hz}), 7.50(1 \mathrm{H}, \mathrm{td}, J=7.6,1.2 \mathrm{~Hz})$, $7.55(1 \mathrm{H}, \mathrm{td}, J=7.6,1.2 \mathrm{~Hz}), 7.80(1 \mathrm{H}, \mathrm{d}, J=7.6 \mathrm{~Hz}), 7.85(1 \mathrm{H}, \mathrm{d}, J=7.6 \mathrm{~Hz}), 7.85(1 \mathrm{H}, \mathrm{d}, J=7.6 \mathrm{~Hz}), 8.20$ $(1 \mathrm{H}, \mathrm{d}, J=7.6 \mathrm{~Hz})$. MS m/z: 440 and $438\left(\mathrm{M}^{+}\right)$. Anal. Calcd for $\mathrm{C}_{27} \mathrm{H}_{19} \mathrm{~N}_{2} \mathrm{O}_{2} \mathrm{Cl} \cdot 1 / 2 \mathrm{EtOAc:} \mathrm{C}, 72.12 ; \mathrm{H}$, 4.80; N, 5.80. Found: C, 71.84; H, 4.68; N, 5.83.

$\left(6 R^{*}, 6 \mathrm{a} R^{*}\right)-6-C h l o r o-12-c y a n o m e t h y l-6 a-h y d r o x y-5,6,6 a, 12-t e t r a h y d r o i n d o l o[2,3-a]$ carbazole-5-one

(8g) from 5 - In the general procedure $\mathrm{A}, \mathrm{K}_{2} \mathrm{CO}_{3}(159 \mathrm{mg}, 1.15 \mathrm{mmol})$, chloroacetonitrile $(0.42 \mathrm{~mL}$, $6.56 \mathrm{mmol}), 5(106 \mathrm{mg}, 0.33 \mathrm{mmol})$, and DMF (3.0 mL) were used. The reaction time was $15 \mathrm{~min}$. After column-chromatography, 8g (72.6 mg, 62\%) was obtained. 8g: $\mathrm{mp} 249.5-251.5^{\circ} \mathrm{C}$ (decomp., yellow prisms, recrystallized from EtOAc). IR (KBr): 3350, 1648, 1615, 1574, 1472, 1345, 1083, 797, 780, 747 $\mathrm{cm}^{-1} .{ }^{1} \mathrm{H}-\mathrm{NMR}\left(\mathrm{DMSO}-d_{6}\right) \delta: 5.41\left(1 \mathrm{H}, \mathrm{d}, J=1.5 \mathrm{~Hz}\right.$, collapsed on addition of $\left.\mathrm{D}_{2} \mathrm{O}\right), 5.99(1 \mathrm{H}, \mathrm{d}, J=18.1$ $\mathrm{Hz}), 6.10(1 \mathrm{H}, \mathrm{d}, J=18.1 \mathrm{~Hz}), 6.95\left(1 \mathrm{H}, \mathrm{d}, J=1.5 \mathrm{~Hz}\right.$, disappeared on addition of $\left.\mathrm{D}_{2} \mathrm{O}\right), 7.42(1 \mathrm{H}, \mathrm{td}$, $J=7.6,1.1 \mathrm{~Hz}), 7.49(1 \mathrm{H}, \mathrm{td}, J=7.6,1.1 \mathrm{~Hz}), 7.57(1 \mathrm{H}, \mathrm{td}, J=7.6,1.1 \mathrm{~Hz}), 7.62(1 \mathrm{H}, \mathrm{td}, J=7.6,1.1 \mathrm{~Hz})$, 
$7.82(1 \mathrm{H}, \mathrm{d}, J=7.6 \mathrm{~Hz}), 7.85(1 \mathrm{H}, \mathrm{d}, J=7.6 \mathrm{~Hz}), 7.95(1 \mathrm{H}, \mathrm{d}, J=7.6 \mathrm{~Hz}), 8.21(1 \mathrm{H}, \mathrm{d}, J=7.6 \mathrm{~Hz})$. Anal. Calcd for $\mathrm{C}_{20} \mathrm{H}_{12} \mathrm{~N}_{3} \mathrm{O}_{2} \mathrm{Cl}$ : C, 66.40; H, 3.34; N, 11.61. Found: C, 66.70; H, 3.33; N, 11.37.

$\left(6 R^{*}, 6 \mathrm{a} R^{*}\right)$-6-Chloro-12- $N, N$-dimethylcarbamoylmethyl-6a-hydroxy-5,6,6a,12-tetrahydroin-

dolo[2,3-a] carbazole-5-one (8h) from 5 - In the general procedure $\mathrm{A}, \mathrm{K}_{2} \mathrm{CO}_{3}(89.2 \mathrm{mg}, 0.65 \mathrm{mmol})$, $N, N$-dimethyl-2-chloroacetamide $(0.66 \mathrm{~mL}, 6.46 \mathrm{mmol}), 5(104 \mathrm{mg}, 0.32 \mathrm{mmol})$, and DMF $(6.0 \mathrm{~mL})$ were used. The reaction time was $1.5 \mathrm{~h}$. After column-chromatography, $\mathbf{8 h}$ (118 $\mathrm{mg}, 90 \%)$ was obtained. 8h: $\mathrm{mp} 257-258^{\circ} \mathrm{C}$ (decomp., dark brown prisms, recrystallized from $\mathrm{MeOH}$ ). IR (KBr): 3410, 1670, 1647, 1583, 1481, 775, $756 \mathrm{~cm}^{-1} .{ }^{1} \mathrm{H}-\mathrm{NMR}$ (DMSO-d $)$ d: $2.90(3 \mathrm{H}, \mathrm{s}), 3.24(3 \mathrm{H}, \mathrm{s}), 5.31(1 \mathrm{H}, \mathrm{s}), 5.64$ $(1 \mathrm{H}, \mathrm{d}, J=16.8 \mathrm{~Hz}), 5.81(1 \mathrm{H}, \mathrm{d}, J=16.8 \mathrm{~Hz}), 6.83\left(1 \mathrm{H}, \mathrm{s}\right.$, disappeared on addition of $\left.\mathrm{D}_{2} \mathrm{O}\right), 7.37(1 \mathrm{H}, \mathrm{td}$, $J=7.5,1.2 \mathrm{~Hz}), 7.39(1 \mathrm{H}, \mathrm{td}, J=7.5,1.2 \mathrm{~Hz}), 7.47(1 \mathrm{H}, \mathrm{td}, J=7.5,1.2 \mathrm{~Hz}), 7.52(1 \mathrm{H}, \mathrm{td}, J=7.5,1.2 \mathrm{~Hz})$, $7.66(1 \mathrm{H}, \mathrm{d}, J=7.5 \mathrm{~Hz}), 7.72(1 \mathrm{H}, \mathrm{d}, J=7.5 \mathrm{~Hz}), 7.82(1 \mathrm{H}, \mathrm{d}, J=7.5 \mathrm{~Hz}), 8.17(1 \mathrm{H}, \mathrm{d}, J=7.5 \mathrm{~Hz}) . \mathrm{MS} m / z$ : 409 and $407\left(\mathrm{M}^{+}\right)$. Anal. Calcd for $\mathrm{C}_{22} \mathrm{H}_{18} \mathrm{~N}_{3} \mathrm{O}_{3} \mathrm{Cl} \cdot 1 / 4 \mathrm{H}_{2} \mathrm{O}: \mathrm{C}, 64.08 ; \mathrm{H}, 4.52 ; \mathrm{N}, 10.19$. Found: C, 64.21; $\mathrm{H}$, $4.52 ; \mathrm{N}, 10.00$.

$\left(6 R^{*}, 6 \mathrm{a} R^{*}\right)-6$-Chloro-6a-hydroxy-12-methoxycarbonylmethyl-5,6,6a,12-tetrahydroindolo[2,3-

a]carbazole-5-one (8i) from 5 - In the general procedure $\mathrm{A}, \mathrm{K}_{2} \mathrm{CO}_{3}(68.2 \mathrm{mg}, 0.48 \mathrm{mmol}$ ), methyl bromoacetate $(0.16 \mathrm{~mL}, 1.61 \mathrm{mmol}), 5(51.8 \mathrm{mg}, 0.16 \mathrm{mmol})$, and DMF $(2.0 \mathrm{~mL})$ were used. The reaction time was $30 \mathrm{~min}$. After column-chromatography, 8i (45.8 $\mathrm{mg}, 72 \%)$ was obtained. 8i: $\mathrm{mp}$ $223-224.5^{\circ} \mathrm{C}$ (decomp., brown prisms, recrystallized from EtOAc). IR (KBr): 3415, 1749, 1655, 1479, 1342, 1086, 1012, 800, 777, $756 \mathrm{~cm}^{-1} .{ }^{1} \mathrm{H}-\mathrm{NMR}$ (DMSO-d $)$ ): $3.74(3 \mathrm{H}, \mathrm{s}), 5.36(1 \mathrm{H}, \mathrm{s}), 5.58(1 \mathrm{H}, \mathrm{d}$, $J=18.0 \mathrm{~Hz}), 5.81(1 \mathrm{H}, \mathrm{d}, J=18.0 \mathrm{~Hz}), 6.84\left(1 \mathrm{H}, \mathrm{s}\right.$, disappeared on addition of $\left.\mathrm{D}_{2} \mathrm{O}\right), 7.39(1 \mathrm{H}, \mathrm{t}, J=7.8$ $\mathrm{Hz}), 7.42(1 \mathrm{H}, \mathrm{t}, J=7.8 \mathrm{~Hz}), 7.48-7.56(2 \mathrm{H}, \mathrm{m}), 7.73(1 \mathrm{H}, \mathrm{d}, J=7.8 \mathrm{~Hz}), 7.79(1 \mathrm{H}, \mathrm{d}, J=7.8 \mathrm{~Hz}), 7.82$ $(1 \mathrm{H}, \mathrm{d}, J=7.8 \mathrm{~Hz}), 8.18(1 \mathrm{H}, \mathrm{d}, J=7.8 \mathrm{~Hz})$. Anal. Calcd for $\mathrm{C}_{21} \mathrm{H}_{15} \mathrm{~N}_{2} \mathrm{O}_{4} \mathrm{Cl}: \mathrm{C}, 63.89 ; \mathrm{H}, 3.83 ; \mathrm{N}, 7.10$. Found: C, 63.65; H, 3.84; N, 6.89 .

$\left(6 R^{*}, 6 \mathrm{a} R^{*}\right)-6$-Chloro-6a-hydroxy-12-[2-(methoxycarbonyl)ethyl]-5,6,6a,12-tetrahydroindolo[2,3a]carbazole-5-one (8j) from 5 - In the general procedure $\mathrm{A}, \mathrm{K}_{2} \mathrm{CO}_{3}(68.7 \mathrm{mg}, 0.47 \mathrm{mmol})$, methyl acrylate $(0.29 \mathrm{~mL}, 3.16 \mathrm{mmol}), \mathbf{5}(50.8 \mathrm{mg}, 0.16 \mathrm{mmol})$, and DMF $(3.0 \mathrm{~mL})$ were used. The reaction time was $30 \mathrm{~min}$. After column-chromatography, 8j (17.0 mg, 26\%) and the unreacted $\mathbf{5}$ (18.5 mg, 36\%) were obtained in the order of elution. 8j: $\mathrm{mp} 216.5-218^{\circ} \mathrm{C}$ (decomp., yellow powder, recrystallized from EtOAc). IR (KBr): 3431, 1714, 1680, 1583, 1479, 1439, 1215, 1146, 773, $754 \mathrm{~cm}^{-1}$. ${ }^{1} \mathrm{H}-\mathrm{NMR}$ (DMSO- $d_{6}$ )

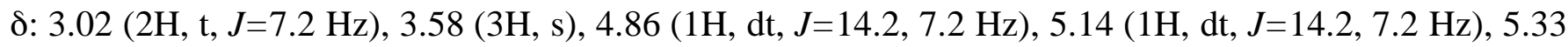
$\left(1 \mathrm{H}, \mathrm{d}, J=1.5\right.$, collapsed on addition of $\left.\mathrm{D}_{2} \mathrm{O}\right), 6.79\left(1 \mathrm{H}, \mathrm{d}, J=1.5\right.$, disappeared on addition of $\left.\mathrm{D}_{2} \mathrm{O}\right), 7.39$ $(1 \mathrm{H}, \mathrm{td}, J=7.7,1.2 \mathrm{~Hz}), 7.40(1 \mathrm{H}, \mathrm{td}, J=7.7,1.2 \mathrm{~Hz}), 7.51(1 \mathrm{H}, \mathrm{td}, J=7.7,1.2 \mathrm{~Hz}), 7.55(1 \mathrm{H}, \mathrm{td}, J=7.7$, $1.2 \mathrm{~Hz}), 7.77(1 \mathrm{H}, \mathrm{d}, J=7.7 \mathrm{~Hz}), 7.83(1 \mathrm{H}, \mathrm{d}, J=7.7 \mathrm{~Hz}), 7.87(1 \mathrm{H}, \mathrm{d}, J=7.7 \mathrm{~Hz}), 8.17(1 \mathrm{H}, \mathrm{d}, J=7.7 \mathrm{~Hz})$. Anal. Calcd for $\mathrm{C}_{22} \mathrm{H}_{17} \mathrm{~N}_{2} \mathrm{O}_{4} \mathrm{Cl}$ : C, 64.59; H, 4.19; N, 6.66. Found: C, 64.63; H, 4.19; N, 6.85. 
$\left(6 R^{*}, 6 \mathrm{a} R^{*}\right)$-6-Chloro-6a-hydroxy-12-phenacyl-5,6,6a,12-tetrahydroindolo[2,3-a]carbazole-5-one

(8k) from 5 - In the general procedure $\mathrm{A}, \mathrm{K}_{2} \mathrm{CO}_{3}(172 \mathrm{mg}, 1.25 \mathrm{mmol})$, phenacyl bromide (1.42 $\mathrm{g}, 7.10$ mmol), 5 (115 mg, $0.33 \mathrm{mmol})$, and DMF (6.0 mL) were used. The reaction time was $1.5 \mathrm{~h}$. After column-chromatography, 8k (152 mg, 97\%) was obtained. 8k: mp 217-219 $\mathrm{C}$ (yellow plates, recrystallized from EtOAc-hexane). IR (KBr): 3372, 1697, 1579, 1475, 1230, $752 \mathrm{~cm}^{-1} .{ }^{1} \mathrm{H}-\mathrm{NMR}$ $\left(\mathrm{DMSO}-d_{6}\right) \delta: 5.36\left(1 \mathrm{H}, \mathrm{d}, J=1.4 \mathrm{~Hz}\right.$, collapsed to s on addition of $\left.\mathrm{D}_{2} \mathrm{O}\right), 6.38(1 \mathrm{H}, \mathrm{d}, J=18.3 \mathrm{~Hz}), 6.52$ $(1 \mathrm{H}, \mathrm{d}, J=18.3 \mathrm{~Hz}), 6.84\left(1 \mathrm{H}, \mathrm{d}, J=1.4 \mathrm{~Hz}\right.$, disappeared on addition of $\left.\mathrm{D}_{2} \mathrm{O}\right), 7.34(1 \mathrm{H}, \mathrm{td}, J=7.6,1.3 \mathrm{~Hz})$, $7.42(1 \mathrm{H}, \mathrm{td}, J=7.6,1.3 \mathrm{~Hz}), 7.45(1 \mathrm{H}, \mathrm{td}, J=7.6,1.3 \mathrm{~Hz}), 7.47(1 \mathrm{H}, \mathrm{td} J=7.6,1.3 \mathrm{~Hz}), 7.51(1 \mathrm{H}, \mathrm{d}, J=8.3$ $\mathrm{Hz}), 7.67(2 \mathrm{H}, \mathrm{t}, J=8.3 \mathrm{~Hz}), 7.76(1 \mathrm{H}, \mathrm{d}, J=7.6 \mathrm{~Hz}), 7.77(1 \mathrm{H}, \mathrm{t}, J=8.3 \mathrm{~Hz}), 7.80(1 \mathrm{H}, \mathrm{d}, J=7.6 \mathrm{~Hz}), 8.17$ $(1 \mathrm{H}, \mathrm{d}, J=7.6 \mathrm{~Hz}), 8.18(1 \mathrm{H}, \mathrm{d}, J=7.6 \mathrm{~Hz}), 8.21(1 \mathrm{H}, \mathrm{d}, J=7.6 \mathrm{~Hz}) . \mathrm{MS} m / z: 442$ and $440\left(\mathrm{M}^{+}\right)$. Anal. Calcd for $\mathrm{C}_{26} \mathrm{H}_{17} \mathrm{~N}_{2} \mathrm{O}_{3} \mathrm{Cl}$ : C, 70.83; H, 3.89; N, 6.35. Found: C, 70.67; H, 3.91; N, 6.24.

$\left(6 R^{*}, 6 a R^{*}\right)-12-(3-B r o m o p r o p y l)-6-c h l o r o-6 a-h y d r o x y-5,6,6 a, 12-t e t r a h y d r o i n d o l o[2,3-a]$ carbazole-

5-one (8I) from 5 - In the general procedure $\mathrm{A}, \mathrm{K}_{2} \mathrm{CO}_{3}(45.7 \mathrm{mg}, 0.33 \mathrm{mmol})$, 1,3-dibromopropane (0.19 mL, $1.89 \mathrm{mmol}), 5(30.5 \mathrm{mg}, 0.09 \mathrm{mmol})$, and DMF $(2.0 \mathrm{~mL})$ were used. The reaction time was 50 min. After column-chromatography, 81 (18.9 mg, 45\%) was obtained. 81: mp 148-150 ${ }^{\circ} \mathrm{C}$ (orange plates, recrystallized from $\mathrm{CHCl}_{3}$ ). IR (KBr): 3400, 1651, 1581, 1479, 1080, $758 \mathrm{~cm}^{-1} .{ }^{1} \mathrm{H}-\mathrm{NMR}\left(\mathrm{CDCl}_{3}\right) \delta$ : $2.51-2.63(2 \mathrm{H}, \mathrm{m}), 3.12\left(1 \mathrm{H}\right.$, br s, disappeared on addition of $\left.\mathrm{D}_{2} \mathrm{O}\right), 3.46(1 \mathrm{H}, \mathrm{ddd}, J=10.4,6.9,5.7 \mathrm{~Hz})$, $3.52(1 \mathrm{H}$, ddd, $J=10.4,6.9,5.7 \mathrm{~Hz}), 4.76(1 \mathrm{H}, \mathrm{s}), 4.80(1 \mathrm{H}, \mathrm{ddd}, J=14.2,7.6,6.5 \mathrm{~Hz}), 5.02(1 \mathrm{H}, \mathrm{dt}$, $J=14.4,7.1 \mathrm{~Hz}), 7.36(1 \mathrm{H}, \mathrm{td}, J=7.8,1.1 \mathrm{~Hz}), 7.41(1 \mathrm{H}, \mathrm{td}, J=7.8,1.1 \mathrm{~Hz}), 7.50(1 \mathrm{H}, \mathrm{td}, J=7.8,1.1 \mathrm{~Hz})$, $7.52(1 \mathrm{H}, \mathrm{td}, J=7.8,1.1 \mathrm{~Hz}), 7.62(1 \mathrm{H}, \mathrm{d}, J=7.8 \mathrm{~Hz}), 7.73(1 \mathrm{H}, \mathrm{d}, J=7.8 \mathrm{~Hz}), 7.92(1 \mathrm{H}, \mathrm{d}, J=7.8 \mathrm{~Hz}), 8.39$ $(1 \mathrm{H}, \mathrm{d}, J=7.8 \mathrm{~Hz})$. HR-MS $\left(\mathrm{FAB}^{+}\right)$: Calcd for $\mathrm{C}_{21} \mathrm{H}_{16} \mathrm{~N}_{2} \mathrm{O}_{2}{ }^{81} \mathrm{Br}^{37} \mathrm{Cl}$ : 447.0112. Found: 447.0084. $\mathrm{C}_{21} \mathrm{H}_{16} \mathrm{~N}_{2} \mathrm{O}_{2}{ }^{79} \mathrm{Br}^{37} \mathrm{Cl}$ : 445.0132. Found: 445.0109. $\mathrm{C}_{21} \mathrm{H}_{16} \mathrm{~N}_{2} \mathrm{O}_{2}{ }^{81} \mathrm{Br}^{35} \mathrm{Cl}$ : 445.0141. Found: 445.0109. $\mathrm{C}_{21} \mathrm{H}_{16} \mathrm{~N}_{2} \mathrm{O}_{2}{ }^{79} \mathrm{Br}{ }^{35} \mathrm{Cl}$ : 443.0162. Found: 443.0132. Anal. Calcd for $\mathrm{C}_{21} \mathrm{H}_{16} \mathrm{~N}_{2} \mathrm{O}_{2} \mathrm{BrCl} \cdot 1 / 4 \mathrm{H}_{2} \mathrm{O}: \mathrm{C}, 56.27 ; \mathrm{H}$, 3.71; N, 6.25. Found: C, 56.33; H, 3.65; N, 5.95.

$\left(6 R^{*}, 6 a R^{*}\right)-6$-Chloro-12-[3-(ethoxycarbonyl)propyl]-6a-hydroxy-5,6,6a,12-tetrahydroindolo[2,3a]carbazole-5-one (8m) from 5 - General Procedure B: A solution of 5 (28.9 mg, $0.09 \mathrm{mmol})$ in anhydrous DMF $(2.0 \mathrm{~mL})$ was added to $60 \% \mathrm{NaH}(3.7 \mathrm{mg}, 0.09 \mathrm{mmol})$ at $0^{\circ} \mathrm{C}$ with stirring under argon atmosphere. After additional stirring at rt, ethyl 4-bromobutylate $(0.26 \mathrm{~mL}, 1.79 \mathrm{mmol})$ was added and the mixture was stirred fot $1 \mathrm{~h}$ at $\mathrm{rt}$. After addition of EtOAc, the whole was washed successively with $\mathrm{H}_{2} \mathrm{O}$, brine, and dried over $\mathrm{Na}_{2} \mathrm{SO}_{4}$, then evaporated under reduced pressure to leave an oil, which was column-chromatographed repeatedly on $\mathrm{SiO}_{2}$ with EtOAc-hexane $(1: 2, \mathrm{v} / \mathrm{v})$ to give $\mathbf{8 m}(23.2 \mathrm{mg}, 59 \%)$ and unreacted 5 (2.80 mg, 10\%) in the order of elution. 8m: (brown viscous oil). IR (film): 3367, 1707, 1672, 1579, 1481, 1200, $750 \mathrm{~cm}^{-1} .{ }^{1} \mathrm{H}-\mathrm{NMR}\left(\mathrm{CDCl}_{3}\right) \delta: 1.15(3 \mathrm{H}, \mathrm{t}, J=7.1 \mathrm{~Hz}), 2.17(2 \mathrm{H}, \mathrm{q}, J=7.1 \mathrm{~Hz})$, $2.38-2.43(2 \mathrm{H}, \mathrm{m}), 4.04(2 \mathrm{H}, \mathrm{q}, J=7.1 \mathrm{~Hz}), 4.70(1 \mathrm{H}, \mathrm{dt}, J=14.2,7.1 \mathrm{~Hz}), 4.91(1 \mathrm{H}, \mathrm{dt}, J=14.2,7.1 \mathrm{~Hz})$, 
$5.32\left(1 \mathrm{H}, \mathrm{d}, J=1.3 \mathrm{~Hz}\right.$, collapsed to s on addition of $\left.\mathrm{D}_{2} \mathrm{O}\right), 6.82(1 \mathrm{H}, \mathrm{d}, J=1.3 \mathrm{~Hz}$, disappeared on addition of $\left.\mathrm{D}_{2} \mathrm{O}\right), 7.39(1 \mathrm{H}, \mathrm{t}, J=7.8 \mathrm{~Hz}), 7.41(1 \mathrm{H}, \mathrm{t}, J=7.8 \mathrm{~Hz}), 7.52(1 \mathrm{H}, \mathrm{td}, J=7.8,1.2 \mathrm{~Hz}), 7.55(1 \mathrm{H}, \mathrm{td}, J=7.8$, $1.2 \mathrm{~Hz}), 7.76(1 \mathrm{H}, \mathrm{d}, J=7.8 \mathrm{~Hz}), 7.84(1 \mathrm{H}, \mathrm{d}, J=7.8 \mathrm{~Hz}), 7.84(1 \mathrm{H}, \mathrm{d}, J=7.8 \mathrm{~Hz}), 8.19(1 \mathrm{H}, \mathrm{d}, J=7.8 \mathrm{~Hz})$. HR-MS $m / z$ : Calcd for $\mathrm{C}_{24} \mathrm{H}_{21} \mathrm{~N}_{2} \mathrm{O}_{4}{ }^{37} \mathrm{Cl}$ : 438.1160. Found: 438.1140. $\mathrm{C}_{24} \mathrm{H}_{21} \mathrm{~N}_{2} \mathrm{O}_{4}{ }^{35} \mathrm{Cl}$ : 436.1189. Found: 436.1184 .

$\left(6 R^{*}, 6 \mathrm{a} R^{*}\right)-6$-Chloro-12-[(Z)-3-chloroallyl]- $(8 \mathrm{n})$ and -12-[(E)-3-chloroallyl]-6a-hydroxy-5,6,6a,12tetrahydroindolo[2,3-a] carbazole-5-one (8o) from 5 - In the general procedure $\mathrm{B}, 5$ (63.5 $\mathrm{mg}, 0.20$ mmol), anhydrous DMF $(4.0 \mathrm{~mL}), 60 \% \mathrm{NaH}(7.90 \mathrm{mg}, 0.20 \mathrm{mmol})$, and $(E, Z)$ mixture of 1,3dichloropropene $(0.36 \mathrm{~mL}, 3.94 \mathrm{mmol})$ were used. After repeated column-chromatography, 8n $(32.9 \mathrm{mg}$, 42\%), 80 (18.4 mg, 24\%), and unreacted 5 (9.10 mg, 14\%) were obtained in the order of elution. 8n: $\mathrm{mp}$ 207-209 ${ }^{\circ} \mathrm{C}$ (decomp., yellow powder, recrystallized from EtOAc). IR (KBr): 3381, 1649, 1616, 1581, 1475, 802, 754, $739 \mathrm{~cm}^{-1} .{ }^{1} \mathrm{H}-\mathrm{NMR}$ (DMSO- $\left.d_{6}\right) \delta: 5.34(1 \mathrm{H}, \mathrm{s}), 5.48$ (1H, ddd, $\left.J=16.1,6.5,2.3 \mathrm{~Hz}\right), 5.73$ $(1 \mathrm{H}, \mathrm{ddd}, J=16.1,6.5,2.3 \mathrm{~Hz}), 6.17(1 \mathrm{H}, \mathrm{q}, J=6.5 \mathrm{~Hz}), 6.63(1 \mathrm{H}, \mathrm{dt}, J=6.5,2.3 \mathrm{~Hz}), 6.84(1 \mathrm{H}$, br s, disappeared on addition of $\left.\mathrm{D}_{2} \mathrm{O}\right), 7.40(1 \mathrm{H}, \mathrm{td}, J=7.6,1.1 \mathrm{~Hz}), 7.43(1 \mathrm{H}, \mathrm{td}, J=7.6,1.1 \mathrm{~Hz}), 7.53(1 \mathrm{H}, \mathrm{td}$, $J=7.6,1.1 \mathrm{~Hz}), 7.55(1 \mathrm{H}, \mathrm{td}, J=7.6,1.1 \mathrm{~Hz}), 7.68(1 \mathrm{H}, \mathrm{d}, J=7.6 \mathrm{~Hz}), 7.78(1 \mathrm{H}, \mathrm{d}, J=7.6 \mathrm{~Hz}), 7.84(1 \mathrm{H}, \mathrm{d}$, $J=7.6 \mathrm{~Hz}), 8.19(1 \mathrm{H}, \mathrm{d}, J=7.6 \mathrm{~Hz}) . \mathrm{MS} m / z: 400,398$, and $396\left(\mathrm{M}^{+}\right)$. Anal. Calcd for $\mathrm{C}_{21} \mathrm{H}_{14} \mathrm{~N}_{2} \mathrm{O}_{2} \mathrm{Cl}_{2} \cdot 1 / 4 \mathrm{H}_{2} \mathrm{O}$ : C, 62.78; H, 3.64; N, 6.97. Found: C, 63.00; H, 3.58; N, 6.95. 8o: brown oil. IR (film): $3417,1653,1577,1471,1146,748 \mathrm{~cm}^{-1} .{ }^{1} \mathrm{H}-\mathrm{NMR}$ (DMSO- $d_{6}$ ) $\delta: 5.34(1 \mathrm{H}, \mathrm{d}, J=1.5 \mathrm{~Hz}$, collapsed to $\mathrm{s}$ on addition of $\left.\mathrm{D}_{2} \mathrm{O}\right), 5.36(1 \mathrm{H}$, ddd, $J=13.4,6.8,1.7 \mathrm{~Hz}), 5.53(1 \mathrm{H}, \mathrm{ddd}, J=13.4,6.8,1.7 \mathrm{~Hz}), 6.30$ $(1 \mathrm{H}, \mathrm{dt}, J=13.4,6.8 \mathrm{~Hz}), 6.74(1 \mathrm{H}, \mathrm{d}, J=13.4 \mathrm{~Hz}), 6.81(1 \mathrm{H}, \mathrm{d}, J=1.5 \mathrm{~Hz}$, disappeared on addition of $\left.\mathrm{D}_{2} \mathrm{O}\right), 7.40(1 \mathrm{H}, \mathrm{td}, J=7.6,1.1 \mathrm{~Hz}), 7.42(1 \mathrm{H}, \mathrm{t}, J=7.6 \mathrm{~Hz}), 7.52(1 \mathrm{H}, \mathrm{td}, J=7.6,1.1 \mathrm{~Hz}), 7.55(1 \mathrm{H}, \mathrm{td}$, $J=7.6,1.1 \mathrm{~Hz}), 7.80(1 \mathrm{H}, \mathrm{d}, J=7.6 \mathrm{~Hz}), 7.84(1 \mathrm{H}, \mathrm{d}, J=7.6 \mathrm{~Hz}), 7.87(1 \mathrm{H}, \mathrm{d}, J=7.6 \mathrm{~Hz}), 8.18(1 \mathrm{H}, \mathrm{d}$, $J=7.6 \mathrm{~Hz}$ ). HR-MS m/z: Calcd for $\mathrm{C}_{21} \mathrm{H}_{14} \mathrm{~N}_{2} \mathrm{O}_{2}{ }^{37} \mathrm{Cl}_{2}$ : 400.0374. Found: 400.0339. $\mathrm{C}_{21} \mathrm{H}_{14} \mathrm{~N}_{2} \mathrm{O}_{2}{ }^{37} \mathrm{Cl}^{35} \mathrm{Cl}$ : 398.0403. Found: 398.0406. $\mathrm{C}_{21} \mathrm{H}_{14} \mathrm{~N}_{2} \mathrm{O}_{2}{ }^{35} \mathrm{Cl}_{2}$ : 396.0433. Found: 396.0449 .

$\left(6 R^{*}, 6 \mathrm{a} R^{*}\right)$-12-Acetyl-6-chloro-6a-hydroxy-5,6,6a,12-tetrahydroindolo[2,3-a] carbazole-5-one

from 5 - In the general procedure B, $5(47.4 \mathrm{mg}, 0.15 \mathrm{mmol})$, anhydrous DMF $(2.0 \mathrm{~mL}), 60 \% \mathrm{NaH}$ $(5.80 \mathrm{mg}, 0.15 \mathrm{mmol})$, and acetyl chloride $(0.21 \mathrm{ml}, 2.94 \mathrm{mmol})$ were used. After repeated columnchromatography, 8p (22.2 mg, 41\%) and unreacted $5(10.9 \mathrm{mg}, 23 \%)$ were obtained in the order of elution. 8p: mp 209-211 ${ }^{\circ} \mathrm{C}$ (decomp., yellow fine needles, recrystallized from EtOAc). IR (KBr): 3332 , 1695, 1685, 1571, 1284, 1263, $760 \mathrm{~cm}^{-1} .{ }^{1} \mathrm{H}-\mathrm{NMR}$ (DMSO- $\left.d_{6}\right) \delta: 2.92(3 \mathrm{H}, \mathrm{s}), 5.31(1 \mathrm{H}, \mathrm{s}), 7.08(1 \mathrm{H}$, br s, disappeared on addition of $\left.\mathrm{D}_{2} \mathrm{O}\right), 7.44(1 \mathrm{H}, \mathrm{td}, J=7.9,1.3 \mathrm{~Hz}), 7.52(1 \mathrm{H}, \mathrm{t}, J=7.9 \mathrm{~Hz}), 7.58(1 \mathrm{H}$, td, $J=7.9,1.3 \mathrm{~Hz}), 7.61(1 \mathrm{H}, \mathrm{td}, J=7.9 \mathrm{~Hz}), 7.80(1 \mathrm{H}, \mathrm{d}, J=7.9 \mathrm{~Hz}), 7.83(1 \mathrm{H}, \mathrm{d}, J=7.9 \mathrm{~Hz}), 8.22(2 \mathrm{H}, \mathrm{d}$, $J=7.9 \mathrm{~Hz}$ ). MS m/z: 366 and $364\left(\mathrm{M}^{+}\right)$. Anal. Calcd for $\mathrm{C}_{20} \mathrm{H}_{13} \mathrm{~N}_{2} \mathrm{O}_{3} \mathrm{Cl} \cdot$ EtOAc: C, 63.65; H, 4.67; N, 6.19. Found: C, 63.42; H, 4.53; N, 6.37. 
12-n-Butyl-6-cyano-5-hydroxyindolo[2,3-a]carbazole (9a) from 8a - General Procedure C: $\mathrm{NaCN}$ (239 mg, $5.72 \mathrm{mmol}$ ) was added to a solution of $\mathbf{8 a}(61.5 \mathrm{mg}, 0.16 \mathrm{mmol})$ in DMF $(4.0 \mathrm{~mL})$ and $\mathrm{H}_{2} \mathrm{O}(2.0$ $\mathrm{mL}$ ), and the mixture was stirred for $0.5 \mathrm{~h}$ at $\mathrm{rt}$. After addition of $\mathrm{H}_{2} \mathrm{O}$, the whole was extracted with EtOAc. The extract was washed with brine, dried over $\mathrm{Na}_{2} \mathrm{SO}_{4}$, and evaporated under reduced pressure to leave an oil, which was column-chromatographed on $\mathrm{SiO}_{2}$ with EtOAc-hexane (1:3, v/v) to give 9a (48.9 mg, 85\%). 9a: mp $251-253^{\circ} \mathrm{C}$ (pale gray powder, recrystallized from EtOAc). IR (KBr): 3311, 2206, 1705, 1630, 1576, 1414, $737 \mathrm{~cm}^{-1} .{ }^{1} \mathrm{H}-\mathrm{NMR}$ (DMSO- $\left.d_{6}\right) \delta: 0.81(3 \mathrm{H}, \mathrm{t}, J=7.4 \mathrm{~Hz}), 1.28(2 \mathrm{H}$, sex, $J=7.4$ $\mathrm{Hz}), 1.82(2 \mathrm{H}$, quin, $J=7.4 \mathrm{~Hz}), 4.86(2 \mathrm{H}, \mathrm{t}, J=7.4 \mathrm{~Hz}), 7.27(1 \mathrm{H}, \mathrm{t}, J=7.7 \mathrm{~Hz}), 7.31(1 \mathrm{H}, \mathrm{t}, J=7.7 \mathrm{~Hz})$, $7.48(1 \mathrm{H}, \mathrm{t}, J=7.7 \mathrm{~Hz}), 7.50(1 \mathrm{H}, \mathrm{t}, J=7.7 \mathrm{~Hz}), 7.72(1 \mathrm{H}, \mathrm{d}, J=7.7 \mathrm{~Hz}), 7.74(1 \mathrm{H}, \mathrm{d}, J=7.7 \mathrm{~Hz}), 8.39(1 \mathrm{H}$, $\mathrm{d}, J=7.7 \mathrm{~Hz}), 8.45(1 \mathrm{H}, \mathrm{d}, J=7.7 \mathrm{~Hz}), 10.7\left(1 \mathrm{H}, \mathrm{s}\right.$, disappeared on addition of $\left.\mathrm{D}_{2} \mathrm{O}\right), 11.5(1 \mathrm{H}, \mathrm{s}$, disappeared on addition of $\left.\mathrm{D}_{2} \mathrm{O}\right)$. MS $m / z: 353\left(\mathrm{M}^{+}\right)$. Anal. Calcd for $\mathrm{C}_{23} \mathrm{H}_{19} \mathrm{~N}_{3} \mathrm{O}: \mathrm{C}, 78.16 ; \mathrm{H}, 5.42 ; \mathrm{N}$, 11.89. Found: C, 77.95; H, 5.47; N, 11.69.

12-Allyl-6-cyano-5-hydroxyindolo[2,3-a] carbazole (9b) from $8 \mathrm{~b}$ - In the general procedure $\mathrm{C}, \mathrm{NaCN}$ (1.50 g, $28.9 \mathrm{mmol}), 8 \mathbf{b}(349 \mathrm{mg}, 0.96 \mathrm{mmol})$, DMF (18.0 mL), and $\mathrm{H}_{2} \mathrm{O}(9.0 \mathrm{~mL})$ were used. After column-chromatography, 9b (294 mg, 91\%) was obtained. 9b: mp 236.5-238 ${ }^{\circ} \mathrm{C}$ (decomp., pale gray cotton fibers, recrystallized from $\mathrm{Et}_{2} \mathrm{O}-$ hexane). IR (KBr): 3440, 2110, 1625, 1458, 1416, 1354, 1168, 916, $736 \mathrm{~cm}^{-1} .{ }^{1} \mathrm{H}-\mathrm{NMR}$ (DMSO- $\left.d_{6}\right) \delta: 4.75(1 \mathrm{H}, \mathrm{d}, J=17.4 \mathrm{~Hz}), 5.06(1 \mathrm{H}, \mathrm{d}, J=10.5 \mathrm{~Hz}), 5.49-5.53(2 \mathrm{H}$, m), 6.10-6.20 (1H, m), $7.26(1 \mathrm{H}, \mathrm{t}, J=7.6 \mathrm{~Hz}), 7.33(1 \mathrm{H}, \mathrm{t}, J=7.6 \mathrm{~Hz}), 7.47(1 \mathrm{H}, \mathrm{t}, J=7.6 \mathrm{~Hz}), 7.49(1 \mathrm{H}$, t, $J=7.6 \mathrm{~Hz}), 7.69(2 \mathrm{H}, \mathrm{d}, J=7.6 \mathrm{~Hz}), 8.39(1 \mathrm{H}, \mathrm{d}, J=7.6 \mathrm{~Hz}), 8.44(1 \mathrm{H}, \mathrm{d}, J=7.6 \mathrm{~Hz}), 10.73(1 \mathrm{H}$, br s, disappeared on addition of $\left.\mathrm{D}_{2} \mathrm{O}\right), 11.53\left(1 \mathrm{H}, \mathrm{s}\right.$, disappeared on addition of $\left.\mathrm{D}_{2} \mathrm{O}\right)$. MS m/z: $337\left(\mathrm{M}^{+}\right)$. Anal. Calcd for $\mathrm{C}_{22} \mathrm{H}_{15} \mathrm{~N}_{3} \mathrm{O}$ : C, 78.32; H, 4.48; N, 12.46. Found: C, 78.08; H, 4.47; N, 12.29.

6-Cyano-5-hydroxy-12-propargylindolo[2,3-a]carbazole (9c) from 8c - In the general procedure C, $\mathrm{NaCN}$ (112 mg, $2.29 \mathrm{mmol}), 8 \mathrm{c}(27.5 \mathrm{mg}, 0.08 \mathrm{mmol})$, DMF (2.0 mL), and $\mathrm{H}_{2} \mathrm{O}(1.0 \mathrm{~mL})$ were used. After column-chromatography, 9c (18.5 mg, 72\%) was obtained. 9c: mp $268-270^{\circ} \mathrm{C}$ (decomp., pale brown powder, recrystallized from $\mathrm{CHCl}_{3}$ ). IR (KBr): 3454, 3263, 2206, 1633, 1460, 1242, $742 \mathrm{~cm}^{-1} .{ }^{1} \mathrm{H}-$ NMR (DMSO- $d_{6}$ ) $\delta: 3.30-3.31\left[1 \mathrm{H}, \mathrm{m}\right.$, clearly appeared at $3.23(1 \mathrm{H}, \mathrm{t}, J=2.2 \mathrm{~Hz})$ on addition of $\left.\mathrm{D}_{2} \mathrm{O}\right]$, $5.75(2 \mathrm{H}, \mathrm{d}, J=2.2 \mathrm{~Hz}), 7.28(1 \mathrm{H}, \mathrm{t}, J=7.8 \mathrm{~Hz}), 7.36(1 \mathrm{H}, \mathrm{t}, J=7.8 \mathrm{~Hz}), 7.49(1 \mathrm{H}, \mathrm{t}, J=7.8 \mathrm{~Hz}), 7.54(1 \mathrm{H}, \mathrm{t}$, $J=7.8 \mathrm{~Hz}), 7.72(1 \mathrm{H}, \mathrm{d}, J=7.8 \mathrm{~Hz}), 7.81(1 \mathrm{H}, \mathrm{d}, J=7.8 \mathrm{~Hz}), 8.39(1 \mathrm{H}, \mathrm{d}, J=7.8 \mathrm{~Hz}), 8.46(1 \mathrm{H}, \mathrm{d}, J=7.8$ $\mathrm{Hz}), 10.8\left(1 \mathrm{H}\right.$, s, disappeared on addition of $\left.\mathrm{D}_{2} \mathrm{O}\right), 11.7\left(1 \mathrm{H}, \mathrm{s}\right.$, disappeared on addition of $\left.\mathrm{D}_{2} \mathrm{O}\right)$. MS $m / z$ : $335\left(\mathrm{M}^{+}\right)$. Anal. Calcd for $\mathrm{C}_{22} \mathrm{H}_{13} \mathrm{~N}_{3} \mathrm{O} \cdot 1 / 2 \mathrm{H}_{2} \mathrm{O}: \mathrm{C}, 76.73 ; \mathrm{H}, 4.10 ; \mathrm{N}, 12.20$. Found: C, 76.86; H, 3.91; N, 11.96 .

12-Benzyl-6-Cyano-5-hydroxyindolo[2,3-a]carbazole (9d) from 8d - In the general procedure C, $\mathrm{NaCN}$ (123 mg, $3.06 \mathrm{mmol}), 8 d$ (32.9 mg, $0.08 \mathrm{mmol})$, DMF (2.0 mL), and $\mathrm{H}_{2} \mathrm{O}(1.0 \mathrm{~mL})$ were used. After column-chromatography, 9d (30.1 mg, 98\%) was obtained. 9d: mp 243-244 ${ }^{\circ} \mathrm{C}$ (decomp., gray 
needles, recrystallized from EtOAc). IR (KBr): 3282, 2200, 1635, 1576, 1169, $739 \mathrm{~cm}^{-1} .{ }^{1} \mathrm{H}-\mathrm{NMR}$ $\left(\mathrm{DMSO}-d_{6}\right) \delta: 6.13(2 \mathrm{H}, \mathrm{s}), 7.14-7.25(5 \mathrm{H}, \mathrm{m}), 7.27(1 \mathrm{H}, \mathrm{td}, J=7.7,1.7 \mathrm{~Hz}), 7.32(1 \mathrm{H}, \mathrm{td}, J=7.7,1.7$ $\mathrm{Hz}), 7.43(1 \mathrm{H}, \mathrm{td}, J=7.7,1.7 \mathrm{~Hz}), 7.46(1 \mathrm{H}, \mathrm{td}, J=7.7,1.7 \mathrm{~Hz}), 7.64(1 \mathrm{H}, \mathrm{d}, J=7.7 \mathrm{~Hz}), 7.65(1 \mathrm{H}, \mathrm{d}, J=7.7$ $\mathrm{Hz}), 8.40(1 \mathrm{H}, \mathrm{d}, J=7.7 \mathrm{~Hz}), 8.46(1 \mathrm{H}, \mathrm{d}, J=7.7 \mathrm{~Hz}), 10.8\left(1 \mathrm{H}\right.$, br s, disappeared on addition of $\left.\mathrm{D}_{2} \mathrm{O}\right)$, $11.7\left(1 \mathrm{H}\right.$, s, disappeared on addition of $\left.\mathrm{D}_{2} \mathrm{O}\right)$. MS m/z: $387\left(\mathrm{M}^{+}\right)$. Anal. Calcd for $\mathrm{C}_{26} \mathrm{H}_{17} \mathrm{~N}_{3} \mathrm{O}: \mathrm{C}, 80.60 ; \mathrm{H}$, 4.42; N, 10.85. Found: C, 80.46; H, 4.47; N, 10.78.

12-Benzyl-6-cyano-5-hydroxyindolo[2,3-a]carbazole (9d) from 13 - Crushed $\mathrm{NaOH}$ powder $(675 \mathrm{mg}$, $16.9 \mathrm{mmol})$ was added to a solution of $13(9.6 \mathrm{mg}, 0.02 \mathrm{mmol})$ in ethylene glycol $(3.0 \mathrm{~mL})$, and the mixture was refluxed for $2 \mathrm{~h}$ with stirring. After addition of $\mathrm{H}_{2} \mathrm{O}$, the whole was extracted with EtOAc. The extract was washed with $\mathrm{H}_{2} \mathrm{O}$ and brine, dried over $\mathrm{Na}_{2} \mathrm{SO}_{4}$, and evaporated under reduced pressure to leave an oil, which was column-chromatographed on $\mathrm{SiO}_{2}$ with EtOAc-hexane (1:3, v/v) to give 9d (5.7 mg, 62\%).

6-Cyano-5-hydroxy-12-phenethylindolo[2,3-a]carbazole (9e) from $8 \mathrm{e}-$ In the general procedure $\mathrm{C}$, $\mathrm{NaCN}$ (117 mg, $2.27 \mathrm{mmol}), 8$ e $(32.3 \mathrm{mg}, 0.08 \mathrm{mmol})$, DMF (2.0 mL), and $\mathrm{H}_{2} \mathrm{O}(1.0 \mathrm{~mL})$ were used. After column-chromatography, 9e (25.8 mg, 85\%) was obtained. 9e: mp 255-257 $\mathrm{C}$ (decomp., pale brown powder, recrystallized from acetone). IR (KBr): 3282, 2212, 1628, 1410, 1238, 742, $700 \mathrm{~cm}^{-1} .{ }^{1} \mathrm{H}-$ NMR (DMSO- $\left.d_{6}\right) \delta: 3.10(2 \mathrm{H}, \mathrm{t}, J=7.2 \mathrm{~Hz}), 5.12(2 \mathrm{H}, \mathrm{t}, J=7.2 \mathrm{~Hz}), 7.04-7.08(1 \mathrm{H}, \mathrm{m}), 7.11(4 \mathrm{H}, \mathrm{d}$, $J=4.4 \mathrm{~Hz}), 7.26(1 \mathrm{H}, \mathrm{t}, J=7.9 \mathrm{~Hz}), 7.27(1 \mathrm{H}, \mathrm{t}, J=7.9 \mathrm{~Hz}), 7.38(1 \mathrm{H}, \mathrm{td}, J=7.9,0.98 \mathrm{~Hz}), 7.48(1 \mathrm{H}, \mathrm{td}$, $J=7.9,0.98 \mathrm{~Hz}), 7.54(1 \mathrm{H}, \mathrm{d}, J=7.9 \mathrm{~Hz}), 7.72(1 \mathrm{H}, \mathrm{d}, J=7.9 \mathrm{~Hz}), 8.34(1 \mathrm{H}, \mathrm{d}, J=7.9 \mathrm{~Hz}), 8.45(1 \mathrm{H}, \mathrm{d}$, $J=7.9 \mathrm{~Hz}), 10.7\left(1 \mathrm{H}\right.$, br s, disappeared on addition of $\left.\mathrm{D}_{2} \mathrm{O}\right), 11.6\left(1 \mathrm{H}, \mathrm{s}\right.$, disappeared on addition of $\left.\mathrm{D}_{2} \mathrm{O}\right)$. MS $m / z: 401\left(\mathrm{M}^{+}\right)$. Anal. Calcd for $\mathrm{C}_{27} \mathrm{H}_{19} \mathrm{~N}_{3} \mathrm{O} \cdot \mathrm{H}_{2} \mathrm{O}: \mathrm{C}, 77.31 ; \mathrm{H}, 5.05 ; \mathrm{N}, 10.02$. Found: C, 77.37; $\mathrm{H}$, $4.85 ; \mathrm{N}, 9.81$.

12-(E)-Cinnamyl-6-Cyano-5-hydroxyindolo[2,3-a] carbazole (9f) from $8 f-$ In the general procedure C, $\mathrm{NaCN}$ (159 mg, $3.23 \mathrm{mmol}), 8 f(47.3 \mathrm{mg}, 0.10 \mathrm{mmol}), \mathrm{DMF}(3.0 \mathrm{~mL})$, and $\mathrm{H}_{2} \mathrm{O}(1.5 \mathrm{~mL})$ were used. After column-chromatography, 9f (35.8 mg, 80\%) was obtained. 9f: yellow viscous oil. IR (film): 3467, 2216, 1631, 1414, 1173, $741 \mathrm{~cm}^{-1} .{ }^{1} \mathrm{H}-\mathrm{NMR}$ (DMSO- $\left.d_{6}\right) \delta: 5.66(2 \mathrm{H}, \mathrm{d}, J=5.1 \mathrm{~Hz}), 6.44(1 \mathrm{H}, \mathrm{d}, J=15.9$ $\mathrm{Hz}), 6.56(1 \mathrm{H}, \mathrm{dt}, J=15.9,5.1 \mathrm{~Hz}), 7.17(1 \mathrm{H}, \mathrm{t}, J=7.4 \mathrm{~Hz}), 7.22(2 \mathrm{H}, \mathrm{t}, J=7.4 \mathrm{~Hz}), 7.26(2 \mathrm{H}, \mathrm{d}, J=7.4 \mathrm{~Hz})$, $7.28(1 \mathrm{H}, \mathrm{t}, J=7.7 \mathrm{~Hz}), 7.34(1 \mathrm{H}, \mathrm{t}, J=7.7 \mathrm{~Hz}), 7.47(1 \mathrm{H}, \mathrm{td}, J=7.7,0.88 \mathrm{~Hz}), 7.50(1 \mathrm{H}, \mathrm{td}, J=7.7,0.88$ Hz), $7.71(1 \mathrm{H}, \mathrm{d}, J=7.7 \mathrm{~Hz}), 7.77(1 \mathrm{H}, \mathrm{d}, J=7.7 \mathrm{~Hz}), 8.41(1 \mathrm{H}, \mathrm{d}, J=7.7 \mathrm{~Hz}), 8.45(1 \mathrm{H}, \mathrm{d}, J=7.7 \mathrm{~Hz}), 10.8$ $\left(1 \mathrm{H}\right.$, br s, disappeared on addition of $\left.\mathrm{D}_{2} \mathrm{O}\right), 11.6\left(1 \mathrm{H}, \mathrm{s}\right.$, disappeared on addition of $\left.\mathrm{D}_{2} \mathrm{O}\right)$. HR-MS $m / z$ : Calcd for $\mathrm{C}_{28} \mathrm{H}_{19} \mathrm{~N}_{3} \mathrm{O}: 413.1528$. Found: 413.1529.

6-Cyano-12-cyanomethyl-5-hydroxyindolo[2,3-a]carbazole $(9 \mathrm{~g})$ from $8 \mathrm{~g}$ - In the general procedure C, $\mathrm{NaCN}$ (1.45 g, $27.8 \mathrm{mmol}), 8 \mathrm{~g}$ (333 mg, $0.93 \mathrm{mmol})$, DMF (24.0 mL), and $\mathrm{H}_{2} \mathrm{O}(12.0 \mathrm{~mL})$ were used. After column-chromatography, 9g (168 mg, 54\%) was obtained. 9g: mp 272.5-275 $\mathrm{C}$ (decomp., pale 
gray powder, recrystallized from EtOAc-hexane). IR (KBr): 3300, 2230, 1630, 1580, 1414, 1320, 1178, 902, $745 \mathrm{~cm}^{-1} .{ }^{1} \mathrm{H}-\mathrm{NMR}\left(\mathrm{DMSO}-d_{6}\right) \delta: 6.17(2 \mathrm{H}, \mathrm{s}), 7.31(1 \mathrm{H}, \mathrm{t}, J=8.0 \mathrm{~Hz}), 7.42(1 \mathrm{H}, \mathrm{t}, J=8.0 \mathrm{~Hz}), 7.53$ $(1 \mathrm{H}, \mathrm{t}, J=8.0 \mathrm{~Hz}), 7.56(1 \mathrm{H}, \mathrm{t}, J=8.0 \mathrm{~Hz}), 7.73(1 \mathrm{H}, \mathrm{d}, J=8.0 \mathrm{~Hz}), 7.88(1 \mathrm{H}, \mathrm{d}, J=8.0 \mathrm{~Hz}), 8.41(1 \mathrm{H}, \mathrm{d}$, $J=8.0 \mathrm{~Hz}), 8.47(1 \mathrm{H}, \mathrm{d}, J=8.0 \mathrm{~Hz}), 10.93\left(1 \mathrm{H}, \mathrm{s}\right.$, disappeared on addition of $\left.\mathrm{D}_{2} \mathrm{O}\right), 11.90(1 \mathrm{H}, \mathrm{s}$, disappeared on addition of $\left.\mathrm{D}_{2} \mathrm{O}\right)$. MS m/z: $336\left(\mathrm{M}^{+}\right)$. Anal. Calcd for $\mathrm{C}_{21} \mathrm{H}_{12} \mathrm{~N}_{4} \mathrm{O} \cdot 1 / 8 \mathrm{H}_{2} \mathrm{O}: \mathrm{C}, 74.49 ; \mathrm{H}$, $3.65 ; \mathrm{N}, 16.55$. Found: C, 74.70; H, 3.62; N, 16.25.

6-Cyano-12- $N, N$-dimethylcarbamoylmethyl-5-hydroxyindolo[2,3-a]carbazole (9h) from $8 \mathrm{~h}-$ In the general procedure C, NaCN (119 mg, $2.43 \mathrm{mmol}), \mathbf{8 h}(33.1 \mathrm{mg}, 0.81 \mathrm{mmol}), \mathrm{DMF}(2.0 \mathrm{~mL})$, and $\mathrm{H}_{2} \mathrm{O}$ $(1.0 \mathrm{~mL})$ were used. After column-chromatography, 9h $(29.6 \mathrm{mg}, 95 \%)$ was obtained. $9 \mathbf{h}: \mathrm{mp}>300^{\circ} \mathrm{C}$ (gray powder, recrystallized from $\mathrm{MeOH}$ ). IR (KBr): 3311, 2216, 1651, 1635, 1412, $742 \mathrm{~cm}^{-1}$. ${ }^{1} \mathrm{H}-\mathrm{NMR}$ $\left(\mathrm{DMSO}-d_{6}\right) \delta: 2.86(3 \mathrm{H}, \mathrm{s}), 3.33(3 \mathrm{H}, \mathrm{s}), 5.75(2 \mathrm{H}, \mathrm{s}), 7.27(1 \mathrm{H}, \mathrm{t}, J=7.8 \mathrm{~Hz}), 7.31(1 \mathrm{H}, \mathrm{t}, J=7.8 \mathrm{~Hz}), 7.45$ $(1 \mathrm{H}, \mathrm{t}, J=7.8 \mathrm{~Hz}), 7.47(1 \mathrm{H}, \mathrm{t}, J=7.8 \mathrm{~Hz}), 7.59(1 \mathrm{H}, \mathrm{d}, J=7.8 \mathrm{~Hz}), 7.67(1 \mathrm{H}, \mathrm{d}, J=7.8 \mathrm{~Hz}), 8.38(1 \mathrm{H}, \mathrm{d}$, $J=7.8 \mathrm{~Hz}), 8.45(1 \mathrm{H}, \mathrm{d}, J=7.8 \mathrm{~Hz}), 10.7\left(1 \mathrm{H}, \mathrm{br}\right.$ s, disappeared on addition of $\left.\mathrm{D}_{2} \mathrm{O}\right), 11.5(1 \mathrm{H}, \mathrm{s}$, disappeared on addition of $\mathrm{D}_{2} \mathrm{O}$ ). HR-MS $m / z$ : Calcd for $\mathrm{C}_{23} \mathrm{H}_{18} \mathrm{~N}_{4} \mathrm{O}_{2}: 382.1430$. Found: 382.1421 .

\section{6-Cyano-5-hydroxy-12-methoxycarbonylmethyl- (9i) and 12-Carboxymethyl-6-cyano-5-}

hydroxyindolo[2,3-a] carbazole (9q) from 8i - In the general procedure C, NaCN (395 mg, 7.64 mmol), 8i (100 mg, $0.25 \mathrm{mmol}), \mathrm{DMF}(4.0 \mathrm{~mL})$, and $\mathrm{H}_{2} \mathrm{O}(2.0 \mathrm{~mL})$ were used. After column-chromatography, 9i $(15.5 \mathrm{mg}, 17 \%)$ was obtained. The aqueous layer was made acidic by adding aq. $8 \% \mathrm{HCl}$ and extracted with EtOAc. The extract was washed with brine, dried over $\mathrm{Na}_{2} \mathrm{SO}_{4}$, and evaporated under reduced pressure to leave a residue, which was column-chromatographed on $\mathrm{SiO}_{2}$ with $\mathrm{CHCl}_{3}-\mathrm{MeOH}-\mathrm{AcOH}$ (46:5:0.5, v/v) to give $9 q(38.6 \mathrm{mg}, 43 \%) .9 \mathbf{i}: \mathrm{mp}>300{ }^{\circ} \mathrm{C}$ (pale gray powder, recrystallized from EtOAc-hexane). IR (KBr): 3410, 2220, 1720, 1630, 1458, 1417, 1240, 1177, $737 \mathrm{~cm}^{-1}$. ${ }^{1} \mathrm{H}-\mathrm{NMR}$ (DMSO$\left.d_{6}\right)$ d: $3.69(3 \mathrm{H}, \mathrm{s}), 5.80(2 \mathrm{H}, \mathrm{s}), 7.28(1 \mathrm{H}, \mathrm{t}, J=7.3 \mathrm{~Hz}), 7.35(1 \mathrm{H}, \mathrm{t}, J=7.3 \mathrm{~Hz}), 7.45-7.52(2 \mathrm{H}, \mathrm{m}), 7.65$ $(1 \mathrm{H}, \mathrm{d}, J=7.3 \mathrm{~Hz}), \quad 7.66(1 \mathrm{H}, \mathrm{d}, J=7.3 \mathrm{~Hz}), 8.39(1 \mathrm{H}, \mathrm{d}, J=7.7 \mathrm{~Hz}), 8.44(1 \mathrm{H}, \mathrm{d}, J=7.7 \mathrm{~Hz}), 10.79(1 \mathrm{H}, \mathrm{s}$, disappeared on addition of $\left.\mathrm{D}_{2} \mathrm{O}\right), 11.65\left(1 \mathrm{H}\right.$, s, disappeared on addition of $\left.\mathrm{D}_{2} \mathrm{O}\right)$. HR-MS $\mathrm{m} / \mathrm{z}$ : Calcd for $\mathrm{C}_{22} \mathrm{H}_{15} \mathrm{~N}_{3} \mathrm{O}_{3}$ : 369.1114. Found: 369.1114. 9q: mp 198-200 ${ }^{\circ} \mathrm{C}$ (decomp., gray powder, recrystallized from MeOH-H $2 \mathrm{O}$ ). IR (KBr): 3367, 2208, 1724, 1631, 1462, 1412, 1323, 1173, 741, $428 \mathrm{~cm}^{-1} .{ }^{1} \mathrm{H}-\mathrm{NMR}$ $\left(\mathrm{DMSO}-d_{6}\right) \delta: 5.64(2 \mathrm{H}, \mathrm{s}), 7.26(1 \mathrm{H}, \mathrm{t}, J=7.8 \mathrm{~Hz}), 7.33(1 \mathrm{H}, \mathrm{t}, J=7.8 \mathrm{~Hz}), 7.41-7.51(2 \mathrm{H}, \mathrm{m}), 7.63(1 \mathrm{H}$, d, $J=8.3 \mathrm{~Hz}), 7.65(1 \mathrm{H}, \mathrm{d}, J=8.3 \mathrm{~Hz}), 8.38(1 \mathrm{H}, \mathrm{d}, J=7.8 \mathrm{~Hz}), 8.43(1 \mathrm{H}, \mathrm{d}, J=7.8 \mathrm{~Hz}), 10.74(1 \mathrm{H}$, br s, disappeared on addition of $\left.\mathrm{D}_{2} \mathrm{O}\right), 11.70\left(1 \mathrm{H}, \mathrm{s}\right.$, disappeared on addition of $\left.\mathrm{D}_{2} \mathrm{O}\right)$. MS m/z: $355\left(\mathrm{M}^{+}\right)$. Anal. Calcd for $\mathrm{C}_{21} \mathrm{H}_{13} \mathrm{~N}_{3} \mathrm{O}_{3} \cdot 1 / 2 \mathrm{H}_{2} \mathrm{O}: \mathrm{C}, 69.22 ; \mathrm{H}, 3.87 ; \mathrm{N}, 11.53$. Found: C, 69.20; H, 3.92; N, 11.27.

\section{2-(3-Bromopropyl)-6-cyano- (9l) and 6-Cyano-12-(3-cyanopropyl)-5-hydroxyindolo[2,3-}

a]carbazole (9r) from 81 - In the general procedure C, $\mathrm{NaCN}$ (107 mg, $2.18 \mathrm{mmol}), 81$ (32.2 mg, 0.07 mmol), DMF (2.0 mL), and $\mathrm{H}_{2} \mathrm{O}(1.0 \mathrm{~mL})$ were used. After column-chromatography, 91 (6.9 mg, 23\%) 
and 9r $(6.1 \mathrm{mg}, 23 \%)$ were obtained in the order of elution. 91: (brown viscous oil). IR (film): 3423, 2210, 1633, 1454, 1246, $746 \mathrm{~cm}^{-1} .{ }^{1} \mathrm{H}-\mathrm{NMR}$ (DMSO-d $)_{6} \delta: 2.40(2 \mathrm{H}, \mathrm{q}, J=6.9 \mathrm{~Hz}), 3.56(2 \mathrm{H}, \mathrm{t}, J=6.9 \mathrm{~Hz}), 4.95$ $(2 \mathrm{H}, \mathrm{t}, J=6.9 \mathrm{~Hz}), 7.28(1 \mathrm{H}, \mathrm{t}, J=7.7 \mathrm{~Hz}), 7.34(1 \mathrm{H}, \mathrm{t}, J=7.7 \mathrm{~Hz}), 7.49(1 \mathrm{H}, \mathrm{td}, J=7.7,1.2 \mathrm{~Hz}), 7.53(1 \mathrm{H}$, td, $J=7.7,1.2 \mathrm{~Hz}), 7.73(1 \mathrm{H}, \mathrm{d}, J=7.7 \mathrm{~Hz}), 7.78(1 \mathrm{H}, \mathrm{d}, J=7.7 \mathrm{~Hz}), 8.40(1 \mathrm{H}, \mathrm{d}, J=7.7 \mathrm{~Hz}), 8.46(1 \mathrm{H}, \mathrm{d}$, $J=7.7 \mathrm{~Hz}), 10.8\left(1 \mathrm{H}\right.$, br s, disappeared on addition of $\left.\mathrm{D}_{2} \mathrm{O}\right), 11.6\left(1 \mathrm{H}\right.$, s, disappeared on addition of $\left.\mathrm{D}_{2} \mathrm{O}\right)$. HR-MS m/z: Calcd for $\mathrm{C}_{22} \mathrm{H}_{16} \mathrm{~N}_{3} \mathrm{O}^{81} \mathrm{Br}$ : 419.0456. Found: 419.0491. $\mathrm{C}_{22} \mathrm{H}_{16} \mathrm{~N}_{3} \mathrm{O}^{79} \mathrm{Br}$ : 417.0477. Found: 417.0451. 9r: (brown viscous oil). IR (film): 3342, 2262, 2210, 1633, 1452, 1400, $756 \mathrm{~cm}^{-1} .{ }^{1} \mathrm{H}-\mathrm{NMR}$ $\left(\mathrm{DMSO}-d_{6}\right) \delta: 2.19(2 \mathrm{H}, \mathrm{q}, J=7.3 \mathrm{~Hz}), 2.61(2 \mathrm{H}, \mathrm{t}, J=7.3 \mathrm{~Hz}), 4.89(2 \mathrm{H}, \mathrm{t}, J=7.3 \mathrm{~Hz}), 7.28(1 \mathrm{H}, \mathrm{t}, J=8.0$ $\mathrm{Hz}), 7.34(1 \mathrm{H}, \mathrm{t}, J=7.7 \mathrm{~Hz}), 7.49(1 \mathrm{H}, \mathrm{t}, J=8.0 \mathrm{~Hz}), 7.53(1 \mathrm{H}, \mathrm{t}, J=8.0 \mathrm{~Hz}), 7.72(1 \mathrm{H}, \mathrm{d}, J=8.0 \mathrm{~Hz}), 7.77$ $(1 \mathrm{H}, \mathrm{d}, J=8.0 \mathrm{~Hz}), 8.40(1 \mathrm{H}, \mathrm{d}, J=8.0 \mathrm{~Hz}), 8.46(1 \mathrm{H}, \mathrm{d}, J=8.0 \mathrm{~Hz}), 10.8(1 \mathrm{H}, \mathrm{s}$, disappeared on addition of $\left.\mathrm{D}_{2} \mathrm{O}\right), 11.6\left(1 \mathrm{H}, \mathrm{s}\right.$, disappeared on addition of $\left.\mathrm{D}_{2} \mathrm{O}\right)$. HR-MS $m / z$ : Calcd for $\mathrm{C}_{23} \mathrm{H}_{16} \mathrm{~N}_{4} \mathrm{O}: 364.1324$. Found: 364.1324.

6-Cyano-12-[3-(ethoxycarbonyl)propyl]-5-hydroxyindolo[2,3-a]carbazole $(9 \mathrm{~m})$ from $8 \mathrm{~m}-$ In the general procedure $\mathrm{C}, \mathrm{NaCN}$ (301 mg, $5.83 \mathrm{mmol}), \mathbf{8 m}$ (84.8 mg, $0.19 \mathrm{mmol}), \mathrm{DMF}(4.0 \mathrm{~mL})$, and $\mathrm{H}_{2} \mathrm{O}$ $(2.0 \mathrm{~mL})$ were used. After column-chromatography, 9m (53.3 mg, 72\%) was obtained. 9m: $\mathrm{mp}$ $258-260^{\circ} \mathrm{C}$ (pale brown powder, recrystallized from $\mathrm{CHCl}_{3}$ ). IR (KBr): 3292, 2210, 1711, 1633, 1242 , 1169, $741 \mathrm{~cm}^{-1} .{ }^{1} \mathrm{H}-\mathrm{NMR}$ (DMSO-d $\left.d_{6}\right) \delta: 1.07(3 \mathrm{H}, \mathrm{t}, J=7.1 \mathrm{~Hz}), 2.13(2 \mathrm{H}$, quin, $J=7.2 \mathrm{~Hz}), 2.41(2 \mathrm{H}, \mathrm{t}$, $J=7.2 \mathrm{~Hz}), 3.95(2 \mathrm{H}, \mathrm{q}, J=7.1 \mathrm{~Hz}), 4.86(2 \mathrm{H}, \mathrm{t}, J=7.2 \mathrm{~Hz}), 7.28(1 \mathrm{H}, \mathrm{t}, J=7.8 \mathrm{~Hz}), 7.33(1 \mathrm{H}, \mathrm{t}, J=7.8 \mathrm{~Hz})$, $7.49(1 \mathrm{H}, \mathrm{td}, J=7.8,1.7 \mathrm{~Hz}), 7.51(1 \mathrm{H}, \mathrm{td}, J=7.8,1.7 \mathrm{~Hz}), 7.72(1 \mathrm{H}, \mathrm{d}, J=7.8 \mathrm{~Hz}), 7.76(1 \mathrm{H}, \mathrm{d}, J=7.8 \mathrm{~Hz})$, $8.39(1 \mathrm{H}, \mathrm{d}, J=7.8 \mathrm{~Hz}), 8.46(1 \mathrm{H}, \mathrm{d}, J=7.8 \mathrm{~Hz}), 10.7\left(1 \mathrm{H}\right.$, br s, disappeared on addition of $\left.\mathrm{D}_{2} \mathrm{O}\right), 11.6(1 \mathrm{H}$, $\mathrm{s}$, disappeared on addition of $\left.\mathrm{D}_{2} \mathrm{O}\right)$. MS $m / z$ : $411\left(\mathrm{M}^{+}\right)$. Anal. Calcd for $\mathrm{C}_{25} \mathrm{H}_{21} \mathrm{~N}_{3} \mathrm{O}_{3} \cdot 1 / 2 \mathrm{H}_{2} \mathrm{O}: \mathrm{C}, 71.41 ; \mathrm{H}$, 5.27; N, 9.99. Found: C, 71.58; H, 5.28; N, 9.78.

\section{(Z)-3-Chloroallyl (9n) and (E)-3-Chloroallyl) 6-cyano-5-hydroxyindolo[2,3-a]carbazole}

(9o) from 8n and 8o - In the general procedure C, $\mathrm{NaCN}$ (208 mg, $4.25 \mathrm{mmol}$ ), about 2:1 mixture of $\mathbf{8 n}$ and $80(56.2 \mathrm{mg}, 0.14 \mathrm{mmol})$, DMF $(3.0 \mathrm{~mL})$, and $\mathrm{H}_{2} \mathrm{O}(1.5 \mathrm{~mL})$ were used. After repeated columnchromatography, 9n $(21.5 \mathrm{mg}, 41 \%)$ and $90(7.3 \mathrm{mg}, 14 \%)$ were obtained in the order of elution. 9n: $>300{ }^{\circ} \mathrm{C}$ (gray powder, recrystallized from EtOAc). IR (KBr): 3454, 2208, 1628, 1412, $742 \mathrm{~cm}^{-1} .{ }^{1} \mathrm{H}-$ NMR (DMSO- $\left.d_{6}\right) \delta: 5.64(2 \mathrm{H}, \mathrm{d}, J=6.6 \mathrm{~Hz}), 6.12(1 \mathrm{H}, \mathrm{q}, J=6.6 \mathrm{~Hz}), 6.58(1 \mathrm{H}, \mathrm{d}, J=6.6 \mathrm{~Hz}), 7.28(1 \mathrm{H}, \mathrm{t}$, $J=7.9 \mathrm{~Hz}), 7.35(1 \mathrm{H}, \mathrm{t}, J=7.9 \mathrm{~Hz}), 7.48(1 \mathrm{H}, \mathrm{t}, J=7.9 \mathrm{~Hz}), 7.52(1 \mathrm{H}, \mathrm{t}, J=7.9 \mathrm{~Hz}), 7.59(1 \mathrm{H}, \mathrm{d}, J=7.9 \mathrm{~Hz})$, $7.70(1 \mathrm{H}, \mathrm{d}, J=7.9 \mathrm{~Hz}), 8.40(1 \mathrm{H}, \mathrm{d}, J=7.9 \mathrm{~Hz}), 8.46(1 \mathrm{H}, \mathrm{d}, J=7.9 \mathrm{~Hz}), 10.8(1 \mathrm{H}$, br s, disappeared on addition of $\left.\mathrm{D}_{2} \mathrm{O}\right), 11.7\left(1 \mathrm{H}, \mathrm{s}\right.$, disappeared on addition of $\left.\mathrm{D}_{2} \mathrm{O}\right)$. HR-MS $\left(\mathrm{FAB}^{+}\right) \mathrm{m} / \mathrm{z}$ : Calcd for $\mathrm{C}_{22} \mathrm{H}_{15} \mathrm{~N}_{3} \mathrm{O}^{37} \mathrm{Cl}$ : 374.0874. Found: 374.0908. $\mathrm{C}_{22} \mathrm{H}_{15} \mathrm{~N}_{3} \mathrm{O}^{35} \mathrm{Cl}$ : 372.0903. Found: 372.0892. 9o: (pale pink viscous oil). IR (film): 3448, 2218, 1630, 1464, 1417, $742 \mathrm{~cm}^{-1} .{ }^{1} \mathrm{H}-\mathrm{NMR}$ (DMSO-d $)$ ) 5.53 (2H, br d, $J=6.3 \mathrm{~Hz}), 6.25(1 \mathrm{H}, \mathrm{dt}, J=12.9,6.3 \mathrm{~Hz}), 6.51(1 \mathrm{H}, \mathrm{d}, J=12.9 \mathrm{~Hz}), 7.28(1 \mathrm{H}, \mathrm{t}, J=7.8 \mathrm{~Hz}), 7.34(1 \mathrm{H}, \mathrm{t}$, 
$J=7.8 \mathrm{~Hz}), 7.48(1 \mathrm{H}, \mathrm{td}, J=7.8,1.7 \mathrm{~Hz}), 7.51(1 \mathrm{H}, \mathrm{td}, J=7.8,1.7 \mathrm{~Hz}), 7.71(1 \mathrm{H}, \mathrm{d}, J=7.8 \mathrm{~Hz}), 7.77(1 \mathrm{H}, \mathrm{d}$, $J=7.8 \mathrm{~Hz}), 8.40(1 \mathrm{H}, \mathrm{d}, J=7.8 \mathrm{~Hz}), 8.45(1 \mathrm{H}, \mathrm{d}, J=7.8 \mathrm{~Hz}), 10.8(1 \mathrm{H}$, br s, disappeared on addition of $\left.\mathrm{D}_{2} \mathrm{O}\right), 11.6\left(1 \mathrm{H}\right.$, br s, disappeared on addition of $\left.\mathrm{D}_{2} \mathrm{O}\right)$. HR-MS $m / z$ : Calcd for $\mathrm{C}_{22} \mathrm{H}_{14} \mathrm{~N}_{3} \mathrm{O}^{37} \mathrm{Cl}$ : 373.0796 . Found: 373.0790. $\mathrm{C}_{22} \mathrm{H}_{14} \mathrm{~N}_{3} \mathrm{O}^{35} \mathrm{Cl}$ : 371.0825.Found: 371.0819 .

6-Cyano-5-hydroxyindolo[2,3-a]carbazole $(10)$ and $\left(6 R^{*}, 6 \mathrm{a} R^{*}, 11 \mathrm{a} R^{*}\right)-6$-chloro-11a-cyano-6ahydroxy-5,6,6a,11,11a,12-hexahydroindolo[2,3-a]carbazole-5-one (11) from 8p - In the general procedure C, $\mathrm{NaCN}$ (109 mg, $2.23 \mathrm{mmol}), 8$ p (27.1 mg, $0.74 \mathrm{mmol})$, DMF (2.0 mL), and $\mathrm{H}_{2} \mathrm{O}(1.0 \mathrm{~mL})$ were used. After repeated column-chromatography with EtOAc-hexane (1:2, v/v) and $\mathrm{CHCl}_{3}-\mathrm{MeOH}$ (99:1, v/v), $10(19.2 \mathrm{mg}, 74 \%)$ and $11(5.6 \mathrm{mg}, 22 \%)$ were obtained in the order of elution. 10: $\mathrm{mp}$ $>300^{\circ} \mathrm{C}$ (pale gray powder, recrystallized from $\mathrm{CHCl}_{3}$ ). IR (KBr): 3373, 2208, 1646, 1569, 1389, 1351, 1324, 1236, $743 \mathrm{~cm}^{-1} .{ }^{1} \mathrm{H}-\mathrm{NMR}$ (DMSO-d $\left.d_{6}\right) \delta: 7.25(1 \mathrm{H}, \mathrm{td}, J=0.9,7.8 \mathrm{~Hz}), 7.28(1 \mathrm{H}, \mathrm{d}, J=7.8 \mathrm{~Hz}), 7.42$ $-7.47(2 \mathrm{H}, \mathrm{m}), 7.73(2 \mathrm{H}, \mathrm{ddd}, J=0.9,5.9,6.7 \mathrm{~Hz}), 8.33(1 \mathrm{H}, \mathrm{d}, J=7.8 \mathrm{~Hz}), 8.39(1 \mathrm{H}, \mathrm{d}, J=7.8 \mathrm{~Hz}), 10.62$ $\left(1 \mathrm{H}\right.$, br s, disappeared on addition of $\left.\mathrm{D}_{2} \mathrm{O}\right), 11.62\left(1 \mathrm{H}\right.$, br s, disappeared on addition of $\left.\mathrm{D}_{2} \mathrm{O}\right), 11.59(1 \mathrm{H}, \mathrm{s}$, disappeared on addition of $\mathrm{D}_{2} \mathrm{O}$ ). Anal. Calcd for $\mathrm{C}_{19} \mathrm{H}_{11} \mathrm{~N}_{3} \mathrm{O}: \mathrm{C}, 76.76 ; \mathrm{H}, 3.73 ; \mathrm{N}, 14.13$. Found: C, 76.81; H, 3.63; N, 14.12. 11: $\mathrm{mp} 231-233^{\circ} \mathrm{C}$ (yellow prisms, recrystallized from $\mathrm{CHCl}_{3}$ ). IR (KBr): 3465 , 2219 (very weak), 1673, 1468, $773 \mathrm{~cm}^{-1} .{ }^{1} \mathrm{H}-\mathrm{NMR}$ (DMSO- $d_{6}$ ) $\delta: 5.17$ (1H, br s, disappeared on addition of $\left.\mathrm{D}_{2} \mathrm{O}\right), 6.79(1 \mathrm{H}, \mathrm{d}, J=7.7 \mathrm{~Hz}), 6.85(1 \mathrm{H}, \mathrm{td}, J=7.7,1.2 \mathrm{~Hz}), 7.20(1 \mathrm{H}, \mathrm{td}, J=7.7,1.2 \mathrm{~Hz}), 7.27(1 \mathrm{H}, \mathrm{td}$, $J=7.7,1.2 \mathrm{~Hz}), 7.35(1 \mathrm{H}, \mathrm{td}, J=7.7,1.2 \mathrm{~Hz}), 7.37\left(1 \mathrm{H}, \mathrm{s}\right.$, disappeared on addition of $\left.\mathrm{D}_{2} \mathrm{O}\right), 7.42(1 \mathrm{H}, \mathrm{s}$, disappeared on addition of $\left.\mathrm{D}_{2} \mathrm{O}\right), 7.47(1 \mathrm{H}, \mathrm{d}, J=7.7 \mathrm{~Hz}), 7.63(1 \mathrm{H}, \mathrm{d}, J=7.7 \mathrm{~Hz}), 7.95(1 \mathrm{H}, \mathrm{d}, J=7.7 \mathrm{~Hz})$, $12.6\left(1 \mathrm{H}\right.$, br s, disappeared on addition of $\left.\mathrm{D}_{2} \mathrm{O}\right)$. HR-MS $\left(\mathrm{FAB}^{+}\right) \mathrm{m} / \mathrm{z}$ : Calcd for $\mathrm{C}_{13} \mathrm{H}_{12} \mathrm{~N}_{3} \mathrm{O}_{2}{ }^{37} \mathrm{Cl}$ : 352.0667 . Found: 352.0701. $\mathrm{C}_{13} \mathrm{H}_{12} \mathrm{~N}_{3} \mathrm{O}_{2}{ }^{35} \mathrm{Cl}$ : 350.697. Found: 350.0703. Anal. Calcd for $\mathrm{C}_{13} \mathrm{H}_{12} \mathrm{~N}_{3} \mathrm{O}_{2} \mathrm{Cl} \cdot 1 / 2 \mathrm{CHCl}_{3}: \mathrm{C}$, 57.20; H, 3.08; N, 10.26. Found: C, 56.94; H, 3.12; N, 9.99.

6-Cyano-5-hydroxyindolo[2,3-a]carbazole (10) from 8k - In the general procedure C, NaCN (11.2 mg, $3.23 \mathrm{mmol}), 8 \mathbf{k}(47.3 \mathrm{mg}, 0.10 \mathrm{mmol})$, DMF (2.0 mL), and $\mathrm{H}_{2} \mathrm{O}(0.1 \mathrm{~mL})$ were used. After columnchromatography, unreacted 8k (5.5 mg, 16\%) and $\mathbf{1 0}(6.1 \mathrm{mg}, 27 \%)$ were obtained in the order of elution. $\left(5 R^{*}, 6 S^{*}, 6 \mathrm{a} R^{*}, 11 \mathrm{a} R^{*}\right)$-6-Chloro-11a-cyano-5,6a-dihydroxy-5,6,6a,11,11a,12-hexahydroindolo[2,3a]carbazole (12a) from $11-\mathrm{NaBH}_{4}(4.5 \mathrm{mg}, 0.12 \mathrm{mmol})$ was added to a solution of 11 (14.1 mg, 0.04 $\mathrm{mmol})$ in $\mathrm{MeOH}(2.0 \mathrm{~mL})$, and the mixture was stirred for $1.5 \mathrm{~h}$ at $\mathrm{rt}$. After addition of $\mathrm{H}_{2} \mathrm{O}$, the whole was extracted with EtOAc. The extract was washed with brine, dried over $\mathrm{Na}_{2} \mathrm{SO}_{4}$, and evaporated under reduced pressure to leave a residue, which was column-chromatographed on $\mathrm{SiO}_{2}$ with EtOAc-hexane $(1: 3, \mathrm{v} / \mathrm{v})$ to give 12a $(6.6 \mathrm{mg}, 47 \%)$ and unreacted $11(7.5 \mathrm{mg}, 53 \%)$ in the order of elution. 12a: yellow viscous oil. IR (film): 3342, 2235 (almost invisible), 1655, 1585, $748 \mathrm{~cm}^{-1} .{ }^{1} \mathrm{H}-\mathrm{NMR}$ (DMSO- $d_{6}$ ) $\delta: 3.93$ $(1 \mathrm{H}, \mathrm{d}, J=8.5 \mathrm{~Hz}), 5.06\left(1 \mathrm{H}, \mathrm{t}, J=8.5 \mathrm{~Hz}\right.$, collapsed to d on addition of $\left.\mathrm{D}_{2} \mathrm{O}\right), 5.72(1 \mathrm{H}, \mathrm{t}, J=8.5 \mathrm{~Hz}$, disappeared on addition of $\left.\mathrm{D}_{2} \mathrm{O}\right), 6.83(1 \mathrm{H}, \mathrm{d}, J=7.5 \mathrm{~Hz}), 6.87(1 \mathrm{H}, \mathrm{td}, J=7.5,1.3 \mathrm{~Hz}), 7.06(1 \mathrm{H}, \mathrm{t}, J=7.5$ 
$\mathrm{Hz}), 7.13\left(1 \mathrm{H}, \mathrm{s}\right.$, disappeared on addition of $\left.\mathrm{D}_{2} \mathrm{O}\right), 7.15\left(1 \mathrm{H}, \mathrm{s}\right.$, disappeared on addition of $\left.\mathrm{D}_{2} \mathrm{O}\right), 7.19(1 \mathrm{H}$, $\mathrm{td}, J=7.5,1.3 \mathrm{~Hz}), 7.21(1 \mathrm{H}, \mathrm{td}, J=7.5,1.3 \mathrm{~Hz}), 7.48(1 \mathrm{H}, \mathrm{d}, J=7.5 \mathrm{~Hz}), 7.51(1 \mathrm{H}, \mathrm{d}, J=7.5 \mathrm{~Hz}), 7.81(1 \mathrm{H}$, $\mathrm{d}, J=7.5 \mathrm{~Hz}), 11.3\left(1 \mathrm{H}, \mathrm{s}\right.$, disappeared on addition of $\left.\mathrm{D}_{2} \mathrm{O}\right)$. HR-MS $\left(\mathrm{FAB}^{+}\right) \mathrm{m} / \mathrm{z}$ : Calcd for $\mathrm{C}_{19} \mathrm{H}_{15} \mathrm{~N}_{3} \mathrm{O}_{2}^{37} \mathrm{Cl}$ : 354.0824. Found: 354.0871. $\mathrm{C}_{19} \mathrm{H}_{15} \mathrm{~N}_{3} \mathrm{O}_{2}{ }^{35} \mathrm{Cl}$ : 352.0853. Found: 352.0846.

$\left(5 R^{*}, 6 S^{*}, 6 \mathrm{a} R *, 11 \mathrm{a} R *\right)-6-C h l o r o-11 \mathrm{a}-c y a n o-5,12-d i a c e t y l-6 a-h y d r o x y-5,6,6 \mathrm{a}, 11,11 \mathrm{a}, 12-$

hexahydroindolo[2,3-a]carbazole $(\mathbf{1 2 b})$ from $12 \mathbf{a}-\mathrm{Ac}_{2} \mathrm{O}(1.0 \mathrm{~mL})$ was added to a solution of $\mathbf{1 2 a}$ $(10.0 \mathrm{mg}, 0.03 \mathrm{mmol})$ in pyridine $(2.0 \mathrm{~mL})$, and the mixture was stirred for $14 \mathrm{~h}$ at $\mathrm{rt}$. After evaporation of the solvent under reduced pressure, the residue was column-chromatographed on $\mathrm{SiO}_{2}$ with EtOAc-hexane (1:3, v/v) to give 12b $(3.7 \mathrm{mg}, 30 \%)$ and unreacted 12a $(5.7 \mathrm{mg}, 57 \%)$ were obtained in the order of elution. 12b: pale yellow oil. IR (film): 3390, 1749, 1705, 1610, 1373, $744 \mathrm{~cm}^{-1} .{ }^{1} \mathrm{H}-\mathrm{NMR}$ $\left(\mathrm{DMSO} d_{6}+5 \% \mathrm{D}_{2} \mathrm{O}, 90^{\circ} \mathrm{C}\right) \delta: 2.13(3 \mathrm{H}, \mathrm{s}), 2.96(3 \mathrm{H}, \mathrm{s}), 4.41(1 \mathrm{H}, \mathrm{d}, J=8.3 \mathrm{~Hz}), 6.56(1 \mathrm{H}, \mathrm{d}, J=8.3 \mathrm{~Hz})$, $6.90(1 \mathrm{H}, \mathrm{td}, J=7.5,1.7 \mathrm{~Hz}), 6.97(1 \mathrm{H}, \mathrm{d}, J=7.5 \mathrm{~Hz}), 7.24(1 \mathrm{H}, \mathrm{td}, J=7.5,1.7 \mathrm{~Hz}), 7.37-7.42(2 \mathrm{H}, \mathrm{m})$, $7.48(1 \mathrm{H}, \mathrm{d}, J=7.5 \mathrm{~Hz}), 7.52(1 \mathrm{H}, \mathrm{td}, J=7.5,1.7 \mathrm{~Hz}), 7.94(1 \mathrm{H}, \mathrm{d}, J=7.5 \mathrm{~Hz})$. HR-MS (FAB $\left.{ }^{+}\right) m / z:$ Calcd for $\mathrm{C}_{23} \mathrm{H}_{19} \mathrm{~N}_{3} \mathrm{O}_{4}{ }^{37} \mathrm{Cl}$ : 438.1035. Found: 438.1040. $\mathrm{C}_{23} \mathrm{H}_{19} \mathrm{~N}_{3} \mathrm{O}_{4}{ }^{35} \mathrm{Cl}$ : 436.1004. Found: 436.1051.

12-Benzyl-6-cyano-5-methoxyindolo[2,3-a]carbazole (13) from 9d - Excess amount of ethereal $\mathrm{CH}_{2} \mathrm{~N}_{2}$ was added to a solution of $9 \mathbf{d}(88.0 \mathrm{mg}, 0.22 \mathrm{mmol})$ in $\mathrm{MeOH}(6.0 \mathrm{~mL})$ and the mixture was stirred for $1.5 \mathrm{~h}$ at $\mathrm{rt}$. The solvent was evaporated under reduced pressure to leave an oil, which was column-chromatographed on $\mathrm{SiO}_{2}$ with $\mathrm{CHCl}_{3}-\mathrm{MeOH}-28 \%$ aq. $\mathrm{NH}_{3}$ (46:0.5:0.05, v/v) to give 13 (75.1 mg, 82\%). 13: mp 228.0—228.5 ${ }^{\circ} \mathrm{C}$ (colorless needles, recrystallized from EtOAc). IR (KBr): 3338, 2208, 1628, 1560, 1390, $741 \mathrm{~cm}^{-1} .{ }^{1} \mathrm{H}-\mathrm{NMR}$ (DMSO- $\left.d_{6}\right) \delta: 4.26(3 \mathrm{H}, \mathrm{s}), 6.16(2 \mathrm{H}, \mathrm{s}), 7.19(2 \mathrm{H}, \mathrm{t}, J=7.7 \mathrm{~Hz})$, $7.20(1 \mathrm{H}, \mathrm{t}, J=7.7 \mathrm{~Hz}), 7.24(1 \mathrm{H}, \mathrm{d}, J=7.7 \mathrm{~Hz}), 7.26(1 \mathrm{H}, \mathrm{d}, J=7.7 \mathrm{~Hz}), 7.33(1 \mathrm{H}, \mathrm{t}, J=7.4 \mathrm{~Hz}), 7.38(1 \mathrm{H}$, t, $J=7.4 \mathrm{~Hz}), 7.50(1 \mathrm{H}, \mathrm{td}, J=7.4,1.9 \mathrm{~Hz}), 7.51(1 \mathrm{H}, \mathrm{td}, J=7.4,1.9 \mathrm{~Hz}), 7.69(1 \mathrm{H}, \mathrm{d}, J=7.4 \mathrm{~Hz}), 7.71(1 \mathrm{H}$, d, $J=7.4 \mathrm{~Hz}), 8.29(1 \mathrm{H}, \mathrm{d}, J=7.4 \mathrm{~Hz}), 8.48(1 \mathrm{H}, \mathrm{d}, J=7.4 \mathrm{~Hz}), 11.9(1 \mathrm{H}, \mathrm{s}$, disappeared on addition of $\left.\mathrm{D}_{2} \mathrm{O}\right)$. MS $m / z$ : $401\left(\mathrm{M}^{+}\right)$. Anal. Calcd for $\mathrm{C}_{27} \mathrm{H}_{19} \mathrm{~N}_{3} \mathrm{O} \cdot 1 / 2 \mathrm{H}_{2} \mathrm{O}: \mathrm{C}, 79.00 ; \mathrm{H}, 4.91 ; \mathrm{N}, 10.24$. Found: $\mathrm{C}$, 79.09; H, 4.90; N, 9.99.

6-(Z)-Aminomethylidene-12-n-butyl-5,6,11,12-tetrahydroindolo[2,3-a]carbazole-5-one (15a) from 9a - General Procedure D: A 1.0 M solution of DIBAL in toluene (2.7 mL, $2.67 \mathrm{mmol})$ was added to a solution of $9 \mathrm{a}(31.4 \mathrm{mg}, 0.09 \mathrm{mmol})$ in anhydrous THF $(2.0 \mathrm{~mL})$ under ice cooling and the mixture was stirred under $\mathrm{N}_{2}$ atmosphere at $\mathrm{rt}$ for $3 \mathrm{~h}$. After addition of $\mathrm{MeOH}$ and aq. Rochelle salt, the whole was extracted with EtOAc. The extract was washed with brine, dried over $\mathrm{Na}_{2} \mathrm{SO}_{4}$, and evaporated under reduced pressure to leave an oil, which was column-chromatographed on $\mathrm{SiO}_{2}$ with $\mathrm{CHCl}_{3}-\mathrm{MeOH}(99: 1$, $\mathrm{v} / \mathrm{v}$ ) to give 15a $(23.0 \mathrm{mg}, 73 \%)$. 15a: $\mathrm{mp} 170-172^{\circ} \mathrm{C}$ (decomp., dark yellow prisms, recrystallized from EtOAc). IR (KBr): 3400, 1628, 1610, 1577, 1560, 1421, $737 \mathrm{~cm}^{-1} .{ }^{1} \mathrm{H}-\mathrm{NMR}\left(\mathrm{DMSO}-d_{6}\right) \delta: 0.82(3 \mathrm{H}, \mathrm{t}$, $J=7.4 \mathrm{~Hz}), 1.29(2 \mathrm{H}$, sex, $J=7.4 \mathrm{~Hz}), 1.81(2 \mathrm{H}$, quin, $J=7.4 \mathrm{~Hz}), 4.77(2 \mathrm{H}, \mathrm{t}, J=7.4 \mathrm{~Hz}), 7.14(1 \mathrm{H}, \mathrm{td}$, 
$J=7.8,1.2 \mathrm{~Hz}), 7.22(1 \mathrm{H}, \mathrm{t}, J=7.8 \mathrm{~Hz}), 7.29(1 \mathrm{H}, \mathrm{t}, J=7.8 \mathrm{~Hz}), 7.31(1 \mathrm{H}, \mathrm{td}, J=7.8,1.2 \mathrm{~Hz}), 7.62(1 \mathrm{H}, \mathrm{d}$, $J=7.8 \mathrm{~Hz}), 7.63(1 \mathrm{H}, \mathrm{d}, J=7.8 \mathrm{~Hz}), 8.02(1 \mathrm{H}, \mathrm{d}, J=7.8 \mathrm{~Hz}), 8.47(1 \mathrm{H}, \mathrm{d}, J=7.8 \mathrm{~Hz}), 8.60(1 \mathrm{H}$, br t,$J=8.2$ $\mathrm{Hz}$, disappeared on addition of $\left.\mathrm{D}_{2} \mathrm{O}\right), 8.84\left(1 \mathrm{H}, \mathrm{dd}, J=13.8,8.2 \mathrm{~Hz}\right.$, collapsed to s on addition of $\left.\mathrm{D}_{2} \mathrm{O}\right)$, $11.1\left(1 \mathrm{H}, \mathrm{s}\right.$, disappeared on addition of $\left.\mathrm{D}_{2} \mathrm{O}\right), 12.0(1 \mathrm{H}, \mathrm{dd}, J=13.8,8.2 \mathrm{~Hz}$, disappeared on addition of $\left.\mathrm{D}_{2} \mathrm{O}\right)$. HR-MS $\mathrm{m} / z$ : Calcd for $\mathrm{C}_{23} \mathrm{H}_{21} \mathrm{~N}_{3} \mathrm{O}$ : 355.1685. Found: 355.1693 .

6-(Z)-Aminomethylidene-12-n-benzyl-5,6,11,12-tetrahydroindolo[2,3-a]carbazole-5-one (15b) from 9d - In the general procedure D, DIBAL (1.4 mL, $1.45 \mathrm{mmol}), 9 d(18.7 \mathrm{mg}, 0.05 \mathrm{mmol})$, THF (2.0 mL) were used. The reaction time was $19 \mathrm{~h}$. After column-chromatography, 15b (6.9 mg, 47\%) was obtained. 15b: mp 207-209 ${ }^{\circ} \mathrm{C}$ (decomp., yellow powder, recrystallized from EtOAc). IR (KBr): 3450, 1628, 1610, 1577, 1560, 1410, $742 \mathrm{~cm}^{-1} .{ }^{1} \mathrm{H}-\mathrm{NMR}$ (DMSO-d $) \delta: 6.04(2 \mathrm{H}, \mathrm{s}), 7.12-7.28(9 \mathrm{H}, \mathrm{m}), 7.53(1 \mathrm{H}, \mathrm{d}, J=7.5$ Hz), $7.54(1 \mathrm{H}, \mathrm{d}, J=7.5 \mathrm{~Hz}), 8.04(1 \mathrm{H}, \mathrm{d}, J=7.5 \mathrm{~Hz}), 8.49(1 \mathrm{H}, \mathrm{dd}, J=7.5,1.7 \mathrm{~Hz}), 8.68(1 \mathrm{H}$, br t,$J=8.4$ $\mathrm{Hz}$, disappeared on addition of $\left.\mathrm{D}_{2} \mathrm{O}\right), 8.88\left(1 \mathrm{H}, \mathrm{dd}, J=13.6,8.4 \mathrm{~Hz}\right.$, collapsed to s on addition of $\left.\mathrm{D}_{2} \mathrm{O}\right)$, $11.2\left(1 \mathrm{H}, \mathrm{s}\right.$, disappeared on addition of $\left.\mathrm{D}_{2} \mathrm{O}\right), 12.1(1 \mathrm{H}, \mathrm{dd}, J=13.6,8.4 \mathrm{~Hz}$, disappeared on addition of $\mathrm{D}_{2} \mathrm{O}$ ). HR-MS $\left(\mathrm{FAB}^{+}\right) \mathrm{m} / \mathrm{z}$ : Calcd for $\mathrm{C}_{26} \mathrm{H}_{20} \mathrm{~N}_{3} \mathrm{O}: 390.1606$. Found: 390.1631 .

6-Acetoaminomethylidene-12-n-butyl-5,6,11,12-tetrahydroindolo[2,3-a]carbazole-5-one (16) from $15 \mathbf{a}-\mathrm{Ac}_{2} \mathrm{O}(0.75 \mathrm{~mL})$ was added to a solution of $\mathbf{1 5 a}(16.1 \mathrm{mg}, 0.05 \mathrm{mmol})$ in pyridine $(1.5 \mathrm{~mL})$, and the mixture was stirred for $1.5 \mathrm{~h}$ at $\mathrm{rt}$. After evaporation of the solvent under reduced pressure, the residue was column-chromatographed on $\mathrm{SiO}_{2}$ successively with EtOAc-hexane (1:3, v/v) and $\mathrm{CHCl}_{3}-\mathrm{MeOH}-28 \%$ aq. $\mathrm{NH}_{3}(46: 0.5: 0.05, \mathrm{v} / \mathrm{v})$ to give 16 (15.7 mg, 87\%). 16: red viscous oil. IR (film): $3354,1685,1620,1610,1552,1415,1273,752,739 \mathrm{~cm}^{-1} .{ }^{1} \mathrm{H}-\mathrm{NMR}\left(\mathrm{CDCl}_{3}\right) \delta: 0.92(3 \mathrm{H}, \mathrm{t}, J=7.5 \mathrm{~Hz})$, $1.37(2 \mathrm{H}$, sex, $J=7.5 \mathrm{~Hz}), 1.81(2 \mathrm{H}$, quin, $J=7.5 \mathrm{~Hz}), 2.28(3 \mathrm{H}, \mathrm{s}), 4.02(2 \mathrm{H}, \mathrm{t}, J=7.5 \mathrm{~Hz}), 7.17(1 \mathrm{H}, \mathrm{dd}$, $J=7.3,1.6 \mathrm{~Hz}), 7.25(1 \mathrm{H}, \mathrm{td}, J=7.3,1.6 \mathrm{~Hz}), 7.30(1 \mathrm{H}, \mathrm{td}, J=7.3,1.6 \mathrm{~Hz}), 7.32(1 \mathrm{H}, \mathrm{td}, J=7.3,1.6 \mathrm{~Hz})$, $7.34(1 \mathrm{H}, \mathrm{td}, J=7.3,1.6 \mathrm{~Hz}), 7.42(1 \mathrm{H}, \mathrm{d}, J=7.3 \mathrm{~Hz}), 7.91(1 \mathrm{H}, \mathrm{d}, J=7.3 \mathrm{~Hz}), 8.24(1 \mathrm{H}, \mathrm{s}$, disappeared on addition of $\left.\mathrm{D}_{2} \mathrm{O}\right), 8.44(1 \mathrm{H}, \mathrm{dd}, J=7.3,1.6 \mathrm{~Hz}), 8.59(1 \mathrm{H}, \mathrm{d}, J=10.4 \mathrm{~Hz}$, collapsed to $\mathrm{s}$ on addition of $\left.\mathrm{D}_{2} \mathrm{O}\right), 13.4\left(1 \mathrm{H}, \mathrm{d}, J=10.4 \mathrm{~Hz}\right.$, disappeared on addition of $\left.\mathrm{D}_{2} \mathrm{O}\right)$. HR-MS $m / z$ : Calcd for $\mathrm{C}_{25} \mathrm{H}_{23} \mathrm{~N}_{3} \mathrm{O}_{2}$ : 397.1791. Found: 397.1790.

X-Ray Crystallographic Analysis of 9a and 11- All measurements were made on a Rigaku AFC5R diffract meter with graphite monochromated $\mathrm{Cu}-K \alpha$ radiation $(\lambda=1.54178 \AA)$. The structure was solved by direct methods using MITHRIL. ${ }^{9}$ Non-hydrogen atoms were refined anisotropically.

9a: a single crystal $(0.20 \times 0.20 \times 0.30 \mathrm{~mm})$ was obtained by recrystallization from EtOAc. Crystal data:

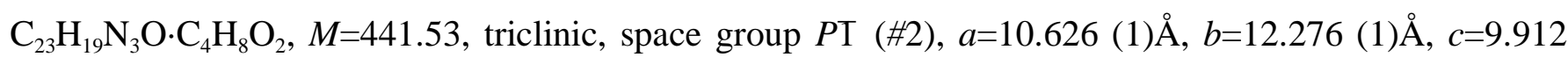

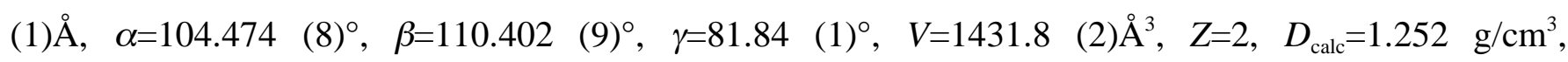
$F(000)=468$, and $\mu(\mathrm{CuK \alpha})=6.26 \mathrm{~cm}^{-1}$. The final cycle of full-matrix least-squares refinement was based on 2807 observed reflections $\left(I>3.00 \sigma(I), 2 \theta<120.2^{\circ}\right)$ and 386 variable parameters. The final 
refinement converged with $R=0.049$ and $R \mathrm{w}=0.059$.

11: a single crystal $(0.20 \times 0.10 \times 0.20 \mathrm{~mm})$ was obtained by recrystallization from $\mathrm{CHCl}_{3}$. $\mathrm{Crystal}$ data:

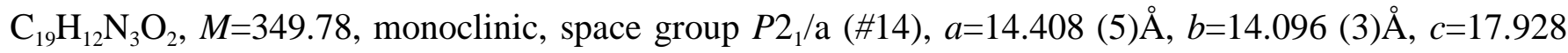
(4) $\AA$, $\beta=93.60(2)^{\circ}, V=3634(2) \AA^{3}, Z=8, D_{\text {calc }}=1.278 \mathrm{~g} / \mathrm{cm}^{3}, F(000)=1440$, and $\mu(\mathrm{Cu} K \alpha)=20.08 \mathrm{~cm}^{-1}$.

The final cycle of full-matrix least-squares refinement was based on 2139 observed reflections $(I>3.00 \sigma$ $\left.(I), 2 \theta<120.4^{\circ}\right)$ and 583 variable parameters. The final refinement converged with $R=0.085$ and $R \mathrm{w}=0.091$.

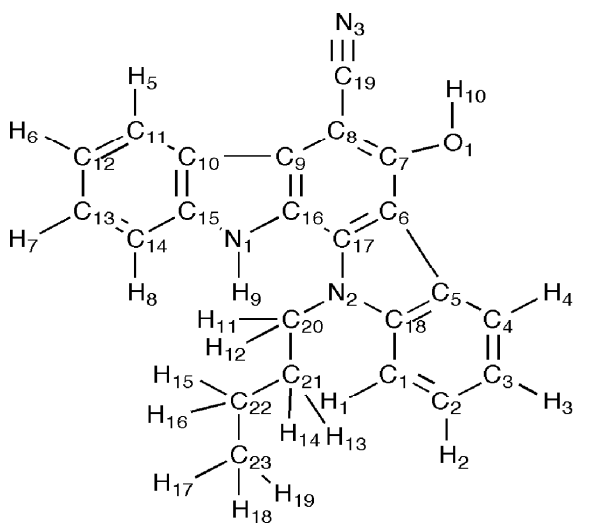<smiles>CC(C)(C)C(=O)OC(C)(C)C(C)(C)C</smiles>

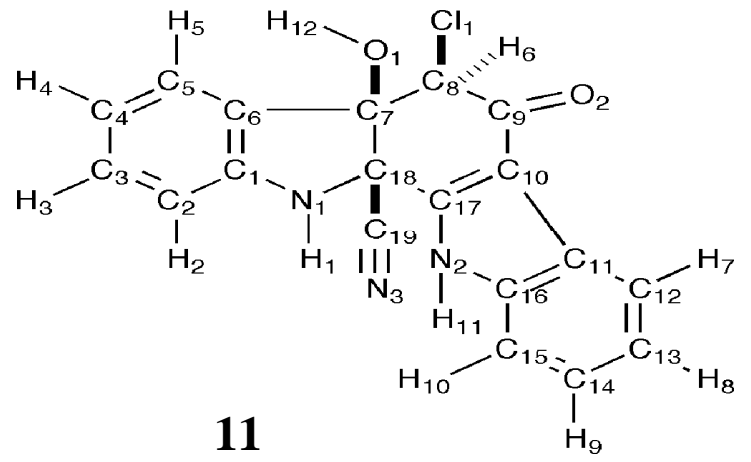

Table 1. Positional parameters and $B(\mathrm{eq})$ for 9 a

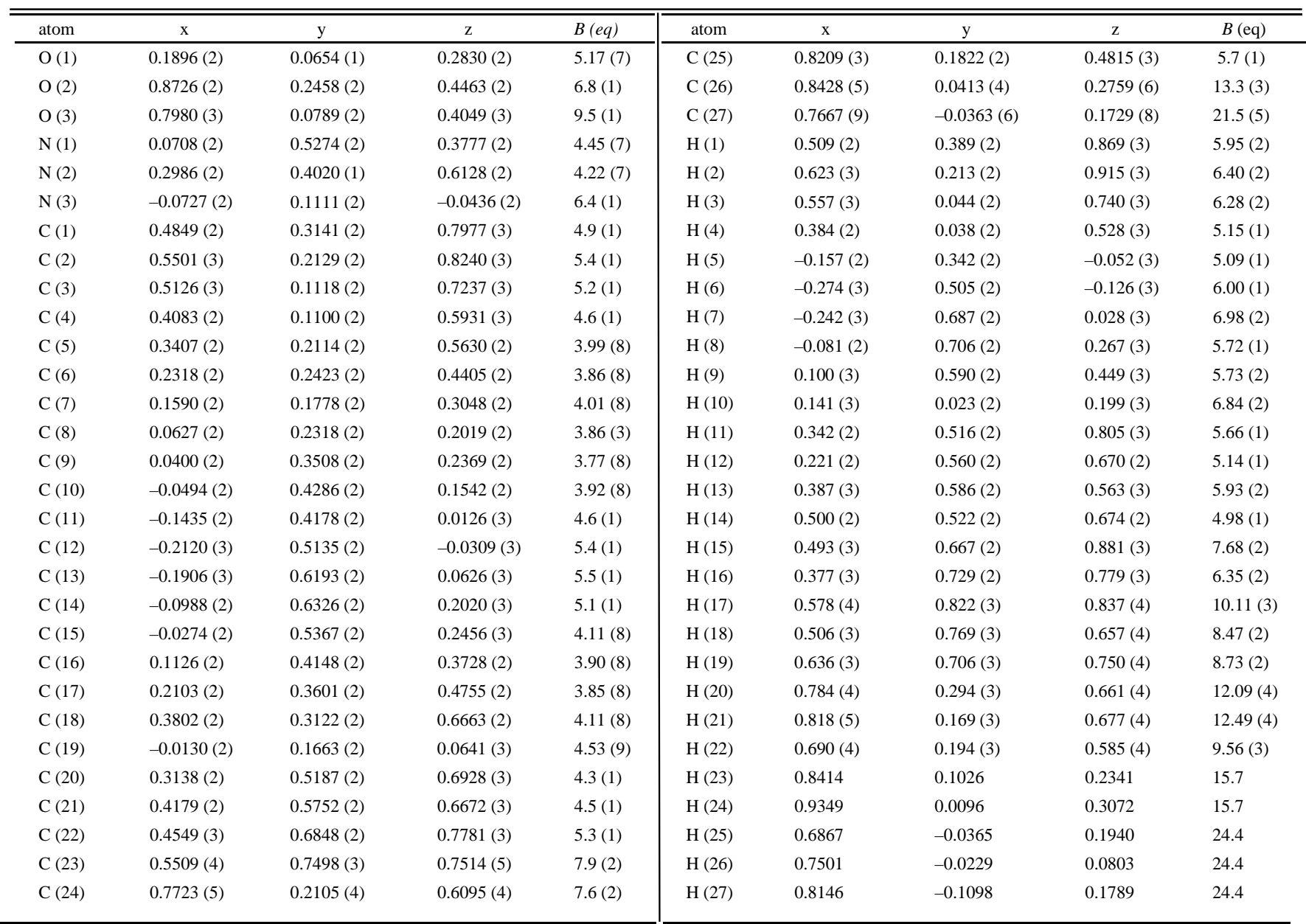


Table 2. Positional parameters and $B(\mathrm{eq})$ for 11

\begin{tabular}{|c|c|c|c|c|c|c|c|c|c|}
\hline atom & $\mathrm{x}$ & $\mathrm{y}$ & $\mathrm{z}$ & $B(e q)$ & atom & $\mathrm{x}$ & $\mathrm{y}$ & $\mathrm{z}$ & $B$ (eq) \\
\hline $\mathrm{O}(1)$ & $0.7922(7)$ & $0.2474(7)$ & $0.6700(7)$ & $3.4(6)$ & C (14) & $0.451(1)$ & $0.322(1)$ & $0.369(1)$ & $6(1)$ \\
\hline $\mathrm{O}(2)$ & $0.7431(8)$ & $0.0971(7)$ & $0.4962(6)$ & $4.4(6)$ & C (15) & $0.503(1)$ & $0.304(2)$ & $0.408(1)$ & $6(1)$ \\
\hline$N(1)$ & $0.870(1)$ & $0.426(1)$ & $0.5746(1)$ & $3.8(8)$ & C (16) & 0.581 (1) & 0.359 (1) & 0.449 (1) & $3.9(9)$ \\
\hline$N(2)$ & $0.648(1)$ & $0.406(1)$ & $0.4915(8)$ & $3.6(8)$ & C (17) & $0.713(1)$ & $0.349(1)$ & $0.5217(8)$ & $2.7(8)$ \\
\hline $\mathrm{N}(3)$ & $0.721(1)$ & 0.479 (1) & $0.6840(9)$ & $5.0(9)$ & C (18) & 0.794 (1) & 0.378 (1) & $0.5773(9)$ & $2.8(8)$ \\
\hline $\mathrm{Cl}(1)$ & $0.9116(3)$ & $0.1120(3)$ & 0.5924 (3) & $5.2(3)$ & C (19) & $0.752(1)$ & $0.434(1)$ & 0.639 (1) & $3.4(9)$ \\
\hline $\mathrm{C}(2)$ & $1.032(2)$ & 0.455 (1) & $0.599(1)$ & $5(1)$ & $\mathrm{H}(2)$ & $0.991(7)$ & $0.247(7)$ & $0.714(6)$ & $0(2)$ \\
\hline C (3) & $1.104(1)$ & $0.418(2)$ & $0.649(1)$ & $5(1)$ & $\mathrm{H}(3)$ & $1.14(1)$ & $0.30(1)$ & $0.725(8)$ & $5(4)$ \\
\hline $\mathrm{C}(4)$ & $1.091(1)$ & 0.339 (1) & $0.692(1)$ & $5(1)$ & H (4) & $1.147(8)$ & $0.450(8)$ & $0.665(6)$ & $0(3)$ \\
\hline C (5) & $1.005(1)$ & 0.295 (1) & 0.685 (1) & $3.3(9)$ & H (5) & $1.044(6)$ & $0.501(6)$ & $0.579(5)$ & $-2(2)$ \\
\hline C (6) & $0.938(1)$ & $0.329(1)$ & $0.635(1)$ & $3.5(9)$ & $\mathrm{H}(6)$ & $0.889(7)$ & $0.230(8)$ & $0.522(6)$ & $1(3)$ \\
\hline C (7) & $0.841(1)$ & $0.206(1)$ & $0.6145(8)$ & $2.5(7)$ & $\mathrm{H}(7)$ & $0.56(1)$ & 0.13 (1) & $0.420(9)$ & $5(5)$ \\
\hline C (8) & 0.847 (1) & $0.212(1)$ & $0.5558(8)$ & $2.7(8)$ & H (8) & $0.444(6)$ & $0.194(6)$ & $0.360(5)$ & $-1(2)$ \\
\hline$C(10)$ & $0.691(1)$ & $0.258(1)$ & $0.4996(9)$ & $2.8(7)$ & $\mathrm{H}(10)$ & 0.49 (1) & $0.442(9)$ & $0.410(8)$ & $2(4)$ \\
\hline C (11) & $0.612(1)$ & $0.263(1)$ & $0.4514(8)$ & $3.6(8)$ & $\mathrm{H}(11)$ & $0.658(7)$ & $0.453(6)$ & $0.497(6)$ & $-2(2)$ \\
\hline C (12) & 0.555 (1) & $0.196(1)$ & $0.413(1)$ & $6(1)$ & $\mathrm{H}(12)$ & $0.804(6)$ & $0.267(6)$ & $0.719(5)$ & $-1(2)$ \\
\hline C (13) & $0.483(1)$ & $0.225(1)$ & $0.372(2)$ & $7(1)$ & & & & & \\
\hline
\end{tabular}

\section{REFERENCES AND NOTES}

1. This report is Part 136 of a series entitled "The Chemistry of Indoles." Part 135: K. Yamada, Y. Tanaka, and M. Somei, Heterocycles, 2009, 79, 635.

2. Review: M. Somei, Heterocycles, 2008, 75, 1021 and references cited therein.

3. M. Somei, F. Yamada, Y. Suzuki, S. Ohmoto, and H. Hayashi, Heterocycles, 2004, 64, 483. H. Hayashi, Y. Suzuki, and M. Somei, Heterocycles, 1999, 51, 1233. H. Hayashi, S. Ohmoto, and M. Somei, Heterocycles, 1997, 45, 1647.

4. G. Knübel, L. K. Larsen, R. E. Moore, I. A. Levine, and G. M. L. Patterson, J. Antibiotics, 1990, 43, 1236.

5. Review of indigo dye: P. E. McGovern and R. H. Michel, Acc. Chem. Res., 1990, 23, 152 and references cited therein.

6. Potent biological activity of $\mathbf{3}$ and its derivatives was reported: T. Ebara, T. Takahashi, and M. Maeda, Abstract of Papers, No. 2, 120th Annual Meeting of Pharmaceutical Society of Japan, Gifu, March, 2000, p. 136.

7. M. Somei, J. Kimura, and S. Takano, JP Patent 4248814 (2009).

8. The invention is now under application for a patent.

9. C. J. Gilmore, J. Appl. Cryst., 1984, 17, 42. 